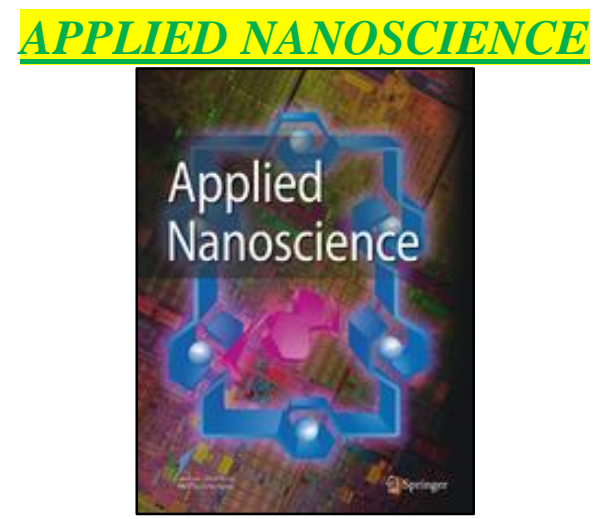

ISSN: $2190-5517$

IMPACT FACTOR: 3.325

PUBLISHER: SPRINGER

ACCEPTED: MARCH 26H, 2018

\title{
EXPERIMENTAL STUDY OF IMPROVED RHEOLOGY AND LUBRICITY OF DRILLING FLUIDS ENHANCED WITH NANO-PARTICLES
}

\author{
O. Anwar Bég1* ${ }^{*}$ D.E. Sanchez Espinoza ${ }^{2}$, Ali Kadir ${ }^{1}$, MD. Shamshuddin ${ }^{* 3}$ and Ayesha Sohail ${ }^{4}$ \\ ${ }^{1}$ Mechanical/Aeronautical Engineering, Salford University, Manchester, M54WT, England, UK. \\ ${ }^{2}$ Petroleum Engineering, 228 Malecón del Salado, Guayacanes y Jiguas, Guayaquil, Ecuador. \\ ${ }^{3}$ Department of Mathematics, Vaagdevi College of Engineering, Warangal, Telangana, India. \\ ${ }^{4}$ Department of Mathematics COMSATS Institute of Information Technology Lahore 54000, Pakistan. \\ *Corresponding author: shammaths@gmail.com,mdshamshuddin@vaagdevieng.ac.in
} ABSTRACT

An experimental study of the rheology and lubricity properties of a drilling fluid is reported, motivated by applications in highly deviated and extended reach wells. Recent developments in nanofluids have identified that the judicious injection of nano-particles into working drilling fluids may resolve a number of issues including borehole instability, lost circulation, torque and drag, pipe sticking problems, bit balling and reduction in drilling speed. The aim of this article is therefore to evaluate the rheological characteristics and lubricity of different nanoparticles in water-based mud, with the potential to reduce costs via a decrease in drag and torque during the construction of highly deviated and ERD wells. Extensive results are presented for percentage in torque variation and coefficient of friction before and after aging. Rheology is evaluated via apparent viscosity, plastic viscosity and gel strength variation before and after aging for water-based muds (WBM). Results are included for silica and titanium nanoparticles at different concentrations. These properties were measured before and after aging the mud samples at $80^{\circ} \mathrm{C}$ during 16 hours at static conditions. The best performance was shown with titanium nanoparticles at a concentration of $0.60 \%(\mathrm{w} / \mathrm{w})$ before aging.

KEY WORDS: Nano-particles; drilling muds; rheology; lubricity; Titanium; Silica; viscosity; gel strength; torque. 


\section{INTRODUCTION}

In the oil and gas industry, drilling is a fundamental and expensive operation. The efficient construction of wells involves many stages including measurement while drilling (MWD), logging while drilling (LWD), directional drilling (DD), casing design, cementing, drilling fluids, solids control and maintenance. A key element of the entire drilling process is drilling fluid engineering which requires carefully fabricated drilling fluid (e.g. muds) which must perform repeatedly many functions at the same time and over long periods (Gray et al. 1980). Drilling fluids (muds) serve to cool and lubricate the drilling bit and other critical features include maintenance of an adequate fluid density, the ability to clean the borehole from drilled cuttings, reducing formation damage (wherein the drilling mud does not achieve sufficient hydrostatic pressure to mitigate formation fluids from entering into the well bore), fluid loss, provision of wellbore stability and creating a mud cake. Although petroleum engineers have developed new methods for due to new challenges in the discovery of new hydrocarbon reserves, there remain problems related to the performance of drilling muds. These include pipe sticking, drag and torque increase, erosion of boreholes, formation consolidation, gel formation and lost circulation. These difficulties, which could delay a continuous operation during drilling, can also result in a huge loss of income for the services companies due to production down time, waste of material and possibly also environmental hazards. Many strategies have been developed in recent years to improve the resilience and in particular lubrication properties of drilling muds. These generally utilize the addition of additives to the drilling mud which is aimed to modify properties and generate enhanced performance. (Dingsøyr et al. 2004) investigated the use of viscosifiers, wetting agents and weighting materials to modify the composition and rheology of drilling fluids. (Dantas et al. 2014) studied experimentally the influence of chemical additives including high-viscosity carboxymethylcellulose (HV-CMC), calcite and starch on rheological and filtration properties of inhibited drilling fluids composed by potassium citrate. They found that fluid loss was minimized for drilling fluids comprising starch and calcite in high concentrations, or with starch, HV-CMC, and calcite in high concentrations. (Qu et al. 2009) presented laboratory test results for the influence of inhibitive property of polyoxyalkyleneamine (POAM) additive in waterbased drilling fluids, demonstrating reduced toxicity, an improvement in the inhibition of hydration of Na-MMT and furthermore a reduction in the swelling or hydration of shale cuttings. (Ahmet et al. 2013) investigated a variety of chemical commercial lubricants on drilling mud (water-based lignosulfonate) rheology and drilling lubricity using a lubricity tester to evaluate the best lubricity/cost ratio of lubricant compositions, noting that the presence of high chloride ion content leads to reduced efficiency in drilling fluid lubricity and rheology. Further studies addressing the modification in drilling fluid lubricity and flow efficiency have been communicated by (Olatunde et al.2012; Behnamanhar et al. 2014; Slawomir et al. 2009) and for polymeric gel additives in drilling fluids (Bu et al. 2013).

In recent years nanotechnology has emerged as a vibrant new development in science and engineering. This involves the manipulation of material properties at the nanoscale. Nanofluids in particular which are synthesized by the suspension of nano-particles in conventional working fluids (water, oil, mud, coolant etc), have yielded considerable improvements in many different applications. Introduced by (Choi, 1995) nanofluids have been shown to dramatically elevate heat transfer properties (such as thermal conductivity, thermal diffusivity) and via modification in viscosity to improve lubrication performance and heat dissipation in friction processes. Nanoparticles have been successfully implemented in medical engineering (Beg et al. 2017), aerospace systems (Kannaiyan et al. 2016), heat exchanger technology (Huminic and Huminic, 2012) and solar energy collectors 
(Kaloudis et al. 2016). With a reduction in fabrication costs and customization of different nano-particles for specific oil fields, nanofluids have recently stimulated some interest in petroleum and gas engineering. (Amanullah and Al-Abdullatif, 2011) showed that spherical nano-particles introduced in drilling muds can decrease differential pipe sticking by forming a thin film around the borehole wall and furthermore may help to dissolve drilling cuttings which aggregate at the base of horizontal well drilling pipes. (Mostafavi et al. 2011) conducted extensive filtration and rheology tests performed on calcium carbonate and iron hydroxide nanoparticles in invert (water/oil) emulsion drilling fluids, noting the significant capabilities of nano calcium carbonate to increase the consistency and sealing capabilities of the filter cakes and iron hydroxide nano-particles in reducing fluid losses. (Ado and Haneef, 2013) reported on the reduction in drilling problems achieved with clay nanoparticles in drilling fluids for deep hydrocarbon wells in Oman. (Al-Yasiri and Al-Sallami, 2015) identified the benefits of nano-particles in overcoming the shortcomings of conventional water and oil-based drilling fluids (gelation, degradation of weighting materials and breakdown of polymeric additives). (Fazelabdolabadi et al. 2015) studied the fabrication of nano-based drilling fluids from water/oil-based fluids using functionalized/unfunctionalized (multi-walled) carbon nanotubes (CNT), showing the influence of volume fraction of CNTs on thermal conductivity, viscosity/yield point, and filtrate amount in all samples. (Zakaria et al. 2012) described a new class of nanoparticle loss circulation materials for oil-based drilling fluids noting a considerable decrease in fluid loss with nano-particles, a thinner filter cake which is beneficial for mitigating formation damage during drilling and also minimizing differential pressure sticking and increased stability of the drilling fluid.

In the current work, results based on a number of rheological and lubricity tests for several different nanoparticles added to water-based mud are described. The objective is to establish whether a decrease in drag and torque is achievable via nanoparticles added to mud which could reduce operational costs in for example highly-deviated wells. Percentage in torque variation before aging and coefficient of friction before aging are documented. Rheological characteristics are computed via apparent viscosity and gel strength variation before aging. It is concluded that improvement in rheological performance can be achieved and that water-based muds (WBMs) may be modified effectively with different nanoparticles for deployment in actual drilling operations. We explore the titanium dioxide and silicon dioxide nano-particles which are used to dope water-based drilling mud. The benefits of these metallic (titanium) and tetravalent metalloid (silicon)-based nano- particles has been well established in many excellent studies in recent years. These materials have been shown to offer considerable advantages in numerous technological applications including biomedical (Yang et al. 2015) and energy systems (Yang et al 2014). Other important studies on nano-particle (e.g. graphene oxide, carbon nanotubes etc) influence on solubility, mechanical properties and adhesion include Lvov et al. (2011), Jin et al. (2014) and Tian et al. (2017). The current study is restricted for brevity to the laboratory analysis of only two nano-particles. However, the methodology is extendable to all of these other advanced nano-materials (and other metallic oxides e.g. zinc oxide, copper oxide, silver oxide etc) indeed efforts in this regard are continuing at Salford University.

\section{METHODOLOGY}

\subsection{Apparatus and Materials}


The potential of nanoparticles in improving drilling mud properties, as testified to by many of the works reviewed in Section 1, have motivated experiments to be conducted in the Salford University Petroleum Engineering Laboratory using silica and titanium nano-particles. These were aimed to evaluate the performance of a water based drilling mud with nanoparticles at different concentrations, in a lubricity tester to determine if these additives could be used as friction reduction agents. Since coefficient of friction is a function of temperature, each sample was heated up at different temperature intervals to analyse its lubricity and rheology before aging, then, the samples were aged during 16 hours in an oven at high temperature to simulate borehole conditions to check for variations in the same parameters. A number of different devices were utilized to quantify properties of the nanoparticles and the drilling muds doped with these particles. A Hamilton Beach high-speed mixer was used to provide a homogeneous mixture of fine particles through their mechanical dispersion at a high shear rate of about 11500 RPM (data retrieved from FANN, 2016). It was used to mix the drilling fluid and nanoparticles. An Ultrasonic Bath - DAWE (Type 6444 Sonicleaner) apparatus which uses ultrasound waves propagated through water was employed to provide a better dispersion of nanoparticles in the WBM. The cavitation phenomenon is used to disperse nanoparticles in the drilling mud since it creates bubbles that explode and help to break the formation of clusters in nanoparticles. This sonicator was used after mixing the nanoparticles in the mixer to prepare the most homogenous mixture possible, as it has been reported that this apparatus reduces the agglomeration of nanoparticles (Hwang et al. 2008). An OFITE mud balance device was used to provide a direct reading of the drilling mud density. It has four scales to measure density that are useful for drilling operations: ppg, SP GR (specific gravity), $l b / f t^{3}$ (pounds per cubic feet), PSI/1000ft (pounds per 1000 feet). The method of operation consists on pouring the drilling fluid into the container, then the lid of the container is placed and some fluid should pass through the hole of the lid. After, the mud balance is placed on the base that support its knife edge and the rider, which acts as a counterweight, is moved across the arm until the bubble of the level vial is in the middle. When equilibrium is achieved, the reading is taken from the different scales. Additionally, its accuracy is $\pm 0.1 \mathrm{ppg}$ or $\pm 0.01 \mathrm{SP}$ GR (data retrieved from OFITE, 2014). An OFITE Model 800 Viscometer, as shown in Figure 1, was used to measure the rheological properties of drilling fluids applying a constant shear rate and shear stress in the drilling mud. This apparatus provides a direct reading of the viscosity, in centipoise (cp), of the drilling mud through a lighted dial. In addition, it has a speed accuracy of \pm 0.1 RPM (data retrieved from OFITE, 2014). Via readings obtained at different speeds, some theological parameters can be calculated such as plastic viscosity, yield point, apparent viscosity and gel strength. The calculations for the first three of these parameters are made with the following formulae:

- $\quad$ Plastic Viscosity (PV):

$P V=600 R P M$ reading $-300 R P M$ reading, $(c p)$

- $\quad$ Yield Point (YP):

$Y P=300 R P M \quad$ reading $-P V,\left(l b / 100 f t^{2}\right)$

- $\quad$ Apparent Viscosity (AV): 
$A P=\frac{600 R P M \text { reading }}{2},(c p)$

\section{- Gel Strength:}

The calculation of the gel strength is taken directly from the dial reading after stopping the apparatus for 10 seconds and 10 minutes, and then turn it on at the speed of 3 RPM (the knob is located on the position of gel). It is measured in $\mathrm{lb} / 100 \mathrm{ft}^{2}$.
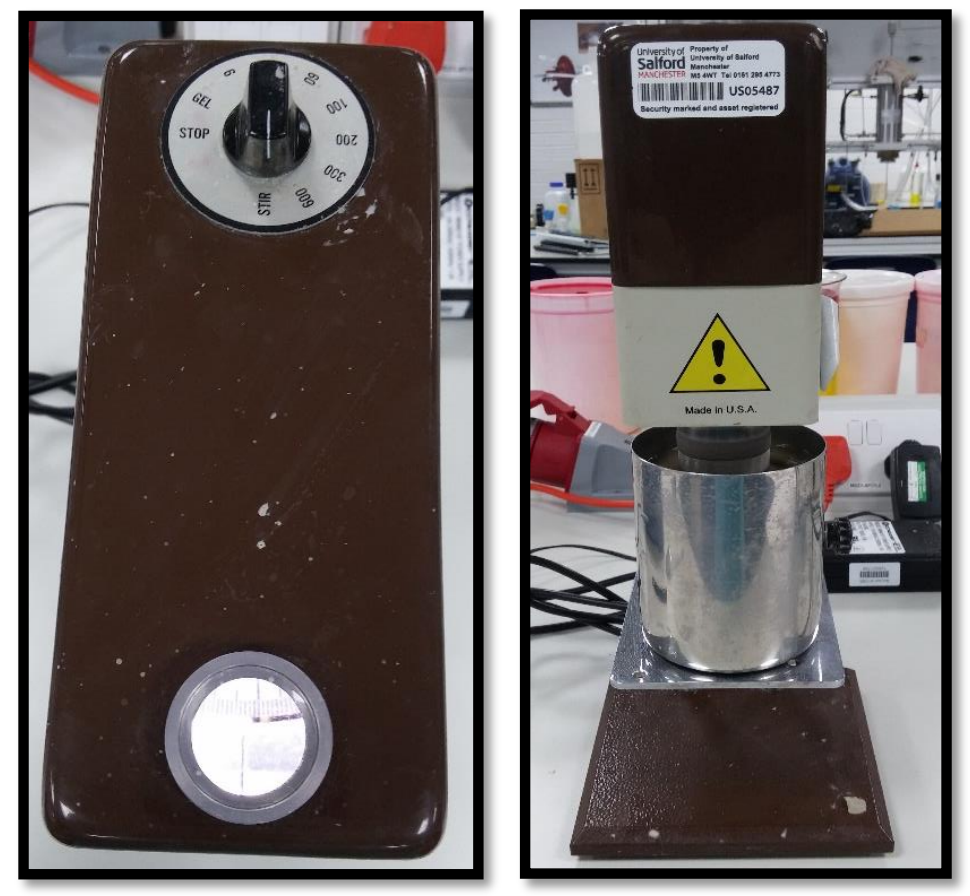

Figure 1: Viscometer - Model 800 OFITE used in nano-particle experiments

An OFITE oven was employed to simulate static or dynamic aging of the drilling mud in borehole conditions at high temperatures and pressures for long periods. This is useful to simulate the behaviour of drilling fluid at the bottom of the borehole, where temperature and pressure are higher, after the drill string has been removed from the well for a long time due to different operations, such as a changing the bit, logging evaluation or placing of a casing section in the well. The oven allows an analysis of the stability of the mud to be performed for realistic working conditions during which the degradation of the fluid is more drastic. A rectification in mud properties may be achieved before running a real operation. The method of operation is simple and it can be programmable. Furthermore, the process of aging is made through an aging cell that keeps the fluid isolated (data retrieved frm OFITE, 2014). An OFITE lubricity Tester (Figure 2) is also deployed to simulate the increase in torque and pressure of a drill string when it is exposed to a high deviation angle during a well construction. During these operations, the drill string tends to settle down at the lower section of the well path. Hence, a section of the drill string could be in contact with the casing, a rock section or drill cuttings, which lead to an increment in the coefficient of friction $(\mathrm{CoF})$ and decreased lubricity, if the lubricating film is broken. 


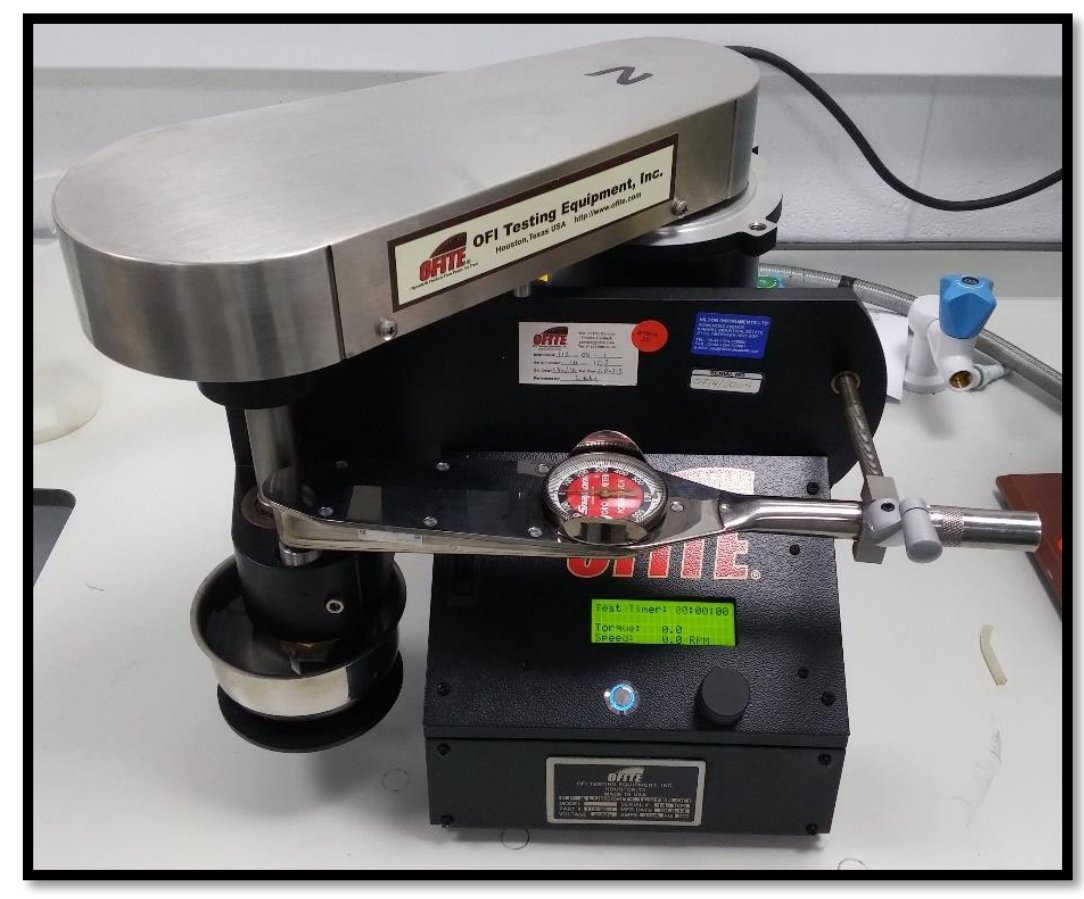

Figure 2: Lubricity Tester (OFI, Houston, Texas, USA)

The lubricity tester measures the lubricity as a function of the $\mathrm{CoF}$ of the drilling mud between two solid steel surfaces: a test ring shaft that turns at a constant speed and the concave groove of a block that is located in a torque shaft lever arm. This lever arm can be adjusted to the desired torque to press both surfaces. The torque arm has a handle that can adjust the torque to a maximum of 600 inch-pounds. Therefore, pressures as high as 100,000 psi can be attained depending of the speed of the ring shaft. The lubricity is measured submerging both, the ring and the block, in a drilling mud sample for a period at a constant speed and torque (of the torque arm). Taking into account that the distance between the centre of the block and torque bushing is 1.5 inches (as it can be observed in figure 3), and the standard test is made at 150 inch-pounds, the resulting force applied is $100 \mathrm{lbf}$. Moreover, a correction factor needs to be applied since the surfaces of both elements are not always the same. Therefore, the $\mathrm{CoF}$ is calculated dividing the meter reading and the force generated by the block when pressed against the test ring using the torque arm.

$$
\text { CoF }=\frac{\text { Meter reading } \times \text { Correction Factor }}{\text { Force of the block against the ring }}
$$

Here:

$$
\text { Correction Factor }=\frac{S \text { tan dered meter reading for Deionized water }}{\text { Meter reading obtained in Deionized water calibration }}
$$

Additionally, an SEM was used to analyse the dispersion, morphology and composition of nanoparticles in the base mud after aging them. Other devices implemented in the experiments included a hotplate stirrer for heating up the drilling mud samples to the desired temperature, a pH meter for controlling the acidity of the mud samples. Nanoparticles utilised for this experiment were titanium oxide $\left(\mathrm{TiO}_{2}\right)$, also known as titanium dioxide or titania, and silicon dioxide $\left(\mathrm{SiO}_{2}\right)$, also known as silica. Properties are summarized in the Appendix. Both nano-particles were provided by US Research Nanomaterials, Inc. These materials occur naturally and have been used for 
numerous different applications. They appear due to the normal process of oxidation of the sources where they are found. In the case of titania which is crystalline, it is found on minerals such as ilmenite, rutile and anatase. The main source of silica is the mineral quartz, although, it can be found in sand as well. Silica is much more abundant than titania. These compounds are categorized as ceramic materials since they are oxides and are therefore hard and brittle. However, the implementation of these materials in drilling muds has not been investigated rigorously and this is a major motivation for the present work, in particular with regard to rheology and lubricity in drilling fluids. The Silicon Dioxide $\left(\mathrm{SiO}_{2}\right)$ nano-particles have an amorphous structure and are coated with 3-4 \% (w/w) KH550 which make them super hydrophilic. The drilling mud used for this experiment was a WBM classified as a Low-Solids Non-Dispersed (LSND). This drilling mud was used to compare results before the addition of nanoparticles. The additives used were bentonite, barite and xanthan gum. Each of these additives performs different functions in this drilling mud. For instance, bentonite is a material made of clay compounds which swells in the presence of water, so that it is normally used to provide efficient hole cleaning and prevent fluid loss into the formation, moreover, is the most common additive used in WBM. Barite is composed by Barium Sulphate and it is utilised as a weighting agent to increase the density of the drilling mud. Xanthan gum is a polymer generated by bacteria and it is used to increase the viscosity of drilling fluids when their density is low.

\subsection{Experimental Procedures}

The following diagram depicts the four stages to the experiments conducted. Firstly, the drilling mud was prepared using the additives mentioned and its rheology and lubricity was evaluated before and after aging. Then, four mud samples were mixed to add two different concentrations of each of the nano-particles and the same tests were performed on them.

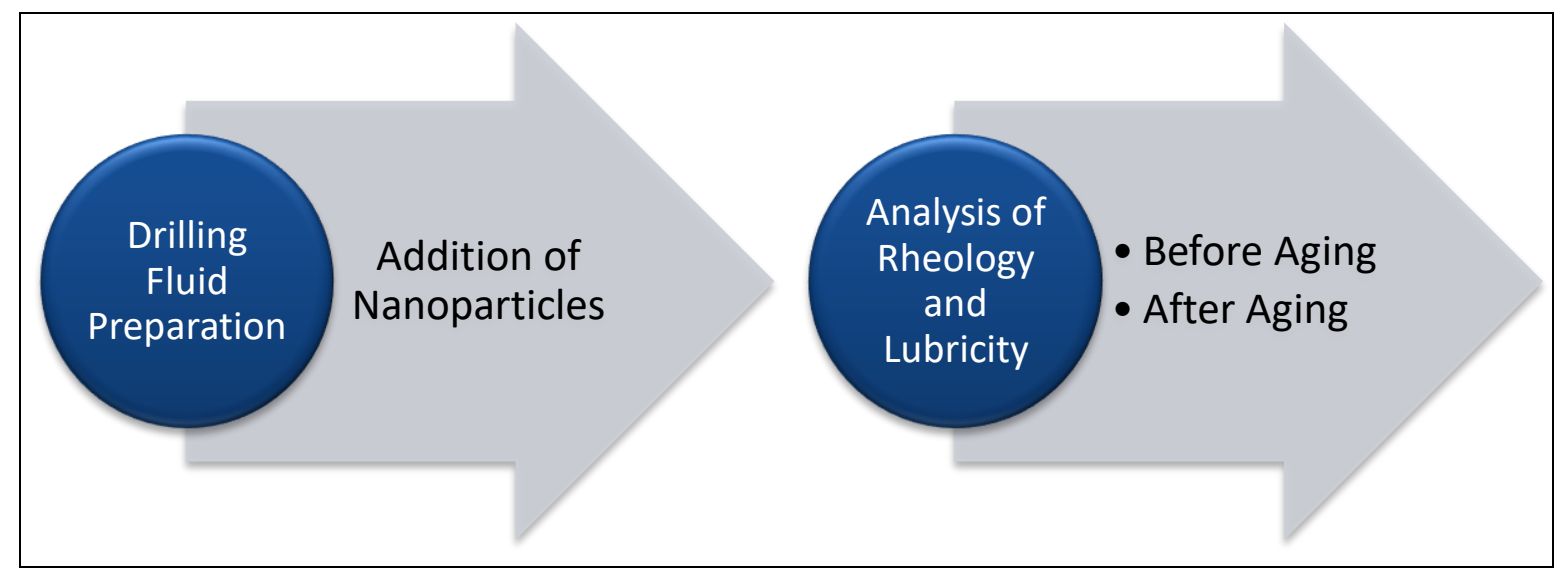

Figure 3: Experimental Procedure

\subsubsection{Drilling Fluid Preparation}

1. In the cup of the mixer were added $350 \mathrm{ml}$ of distilled water and 15 grams of bentonite. This mixture was stirred for 15 minutes approximately. It was then left at rest for the same amount of time to allow clay hydration.

2. Next 15 grams of Barite were added to increase the density of the fluid, and it was mixed for five minutes. 
3. Finally, 0.7 grams of xanthan gum were added in small portions to avoid the formation of lumps in the drilling mud and produce a more homogeneous mixture. This was stirred for 10 minutes and left at rest for the same amount of time.

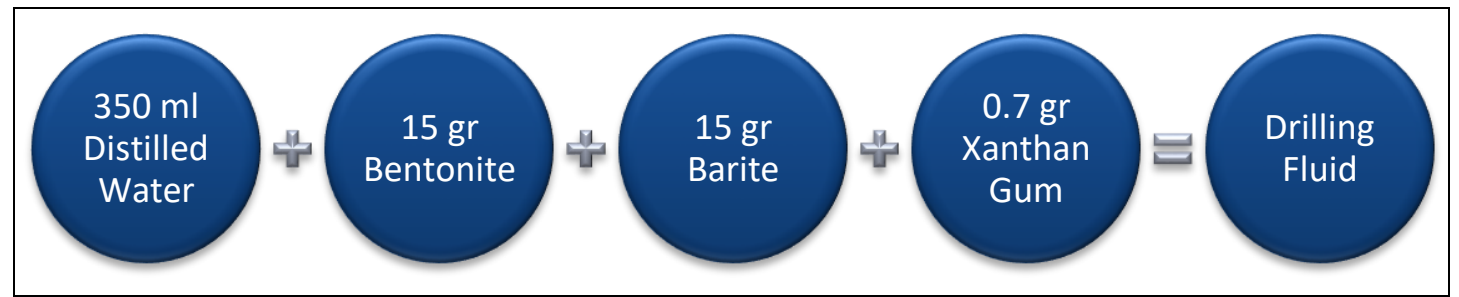

Figure 4: Drilling Fluid Preparation

\subsubsection{Addition of Nanoparticles}

1. After preparing the drilling fluid and left at rest for some time, nanoparticles were added to the drilling mud in the following concentrations (Table 1) for each mud sample of $350 \mathrm{ml}$ :

2. Then, the nanoparticles were mixed in the drilling fluid using two different two-step methods: stirring and an ultrasonic agitation.

3. When using the mixer, nanoparticles were stirred at high speed during 40 minutes.

4. Soon after, they were introduced in the ultrasonic bath using Erlenmeyer flasks, as it can be observed in the following figure:

5. After 30 minutes, the ultrasonic bath was stopped and the samples were left to rest at room temperature.

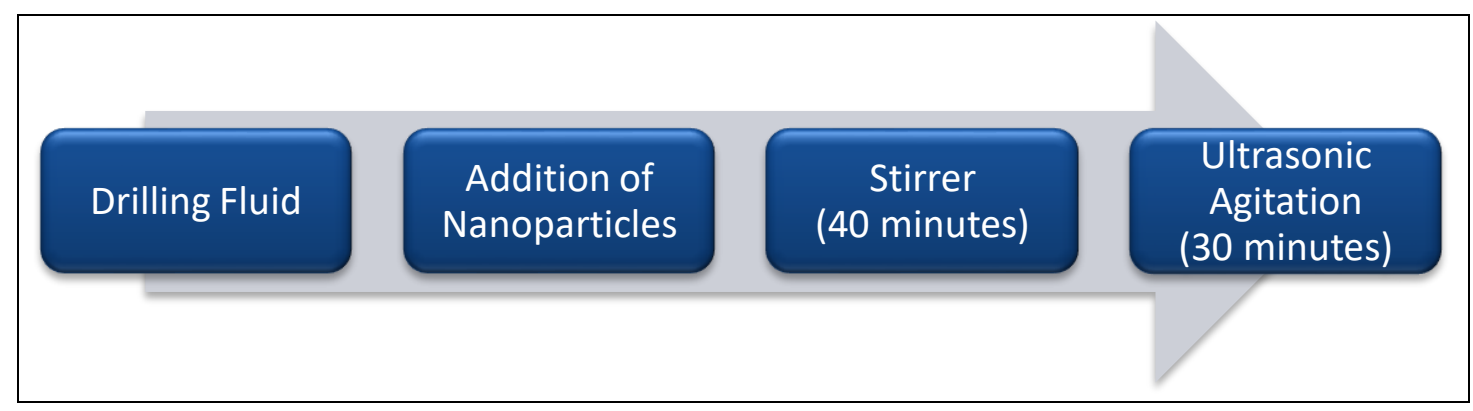

Figure 5: Addition of nanoparticles in the drilling fluid

Table 1: Nano-particle concentrations

\begin{tabular}{|c|c|}
\hline Nanoparticles & Concentration (w/w \%) \\
\hline \multirow{2}{*}{$\mathrm{Ti}_{2}$} & 0.20 \\
\cline { 2 - 2 } & 0.60 \\
\hline \multirow{2}{*}{$\mathrm{SiO}_{2}$} & 0.20 \\
\hline & 0.60 \\
\hline
\end{tabular}




\subsubsection{Analysis of Rheology and Lubricity before Aging}

Drilling fluid samples before and after adding nanoparticles were evaluated in the same way.

1. After drilling fluid samples were cooled down to $26^{\circ} \mathrm{C}$, density, rheology and lubricity was measured using the mud balance, viscometer and lubricity tester, respectively.

2. The rheology was measured observing a stable value in the dial reading at 600,300, 200, 100, 60, 30 and 6 RPM. Gel strength was measured observing the maximum deflection in the dial reading at 3 RPM when the viscometer was turned on at this speed after the mud sample was left at rest for 10 seconds and 10 minutes.

3. The lubricity test was carried out calculating the correction factor for the lubricity ring and lubricity block and observed each minute during 6 minutes.

4. Then, the fluid samples were heated up in the hotplate stirrer at 40,60 and 80 degrees Celsius $\left({ }^{\circ} \mathrm{C}\right)$, and the same tests were performed to simulate the mud samples behaviour during drilling operations.

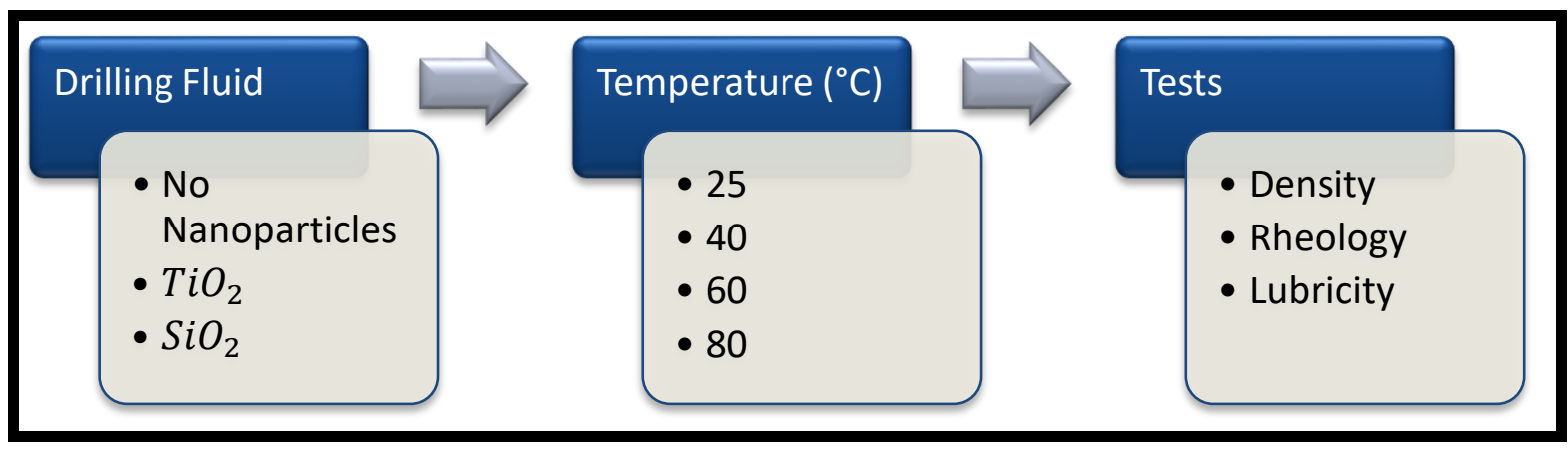

Figure 6: Analysis performed before aging

\subsubsection{Analysis of Rheology and Lubricity after Aging}

1. The drilling fluid samples (with and without nanoparticles) were poured into aging cells.

2. Then, they were placed in the oven for 16 hours at $80{ }^{\circ} \mathrm{C}$. The samples remained static during this period to simulate bottom hole conditions.

3. After this time, the samples were cooled down to $26{ }^{\circ} \mathrm{C}$ and its rheology was evaluated. Then, the samples were stirred in the mixer for 10 minutes and its rheology was tested again, as well as its lubricity using the same procedure for the samples before aging.

In addition, during the drilling fluid preparation, the $\mathrm{pH}$ level of the mixture was continuously checked with the $\mathrm{pH}$ meter to maintain it at 10 via the addition of caustic soda. 

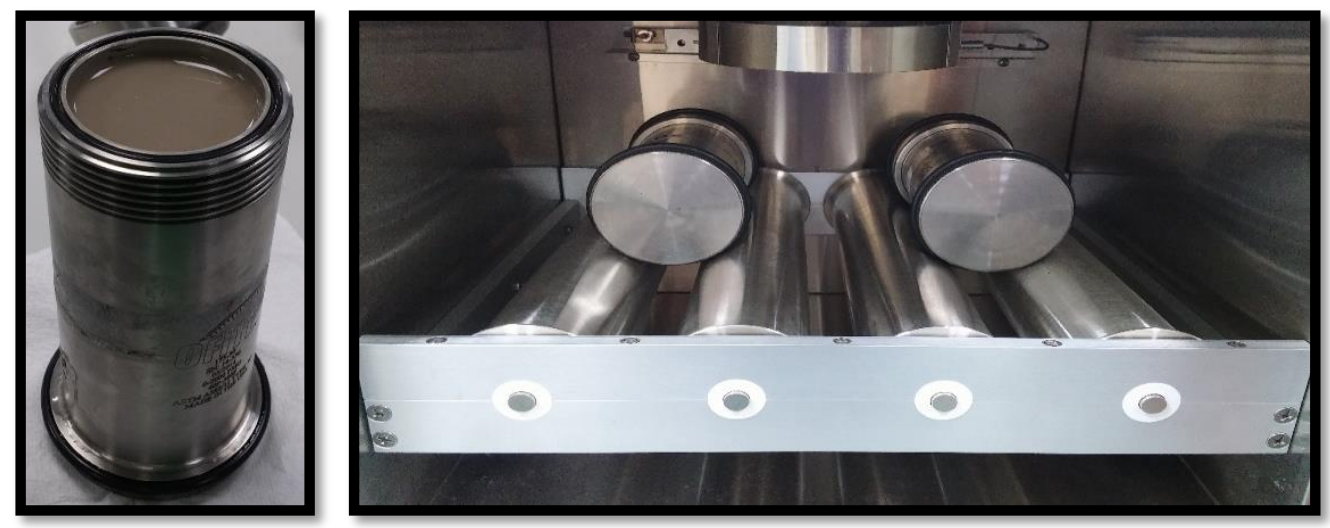

Figure 7: Aging cells and oven

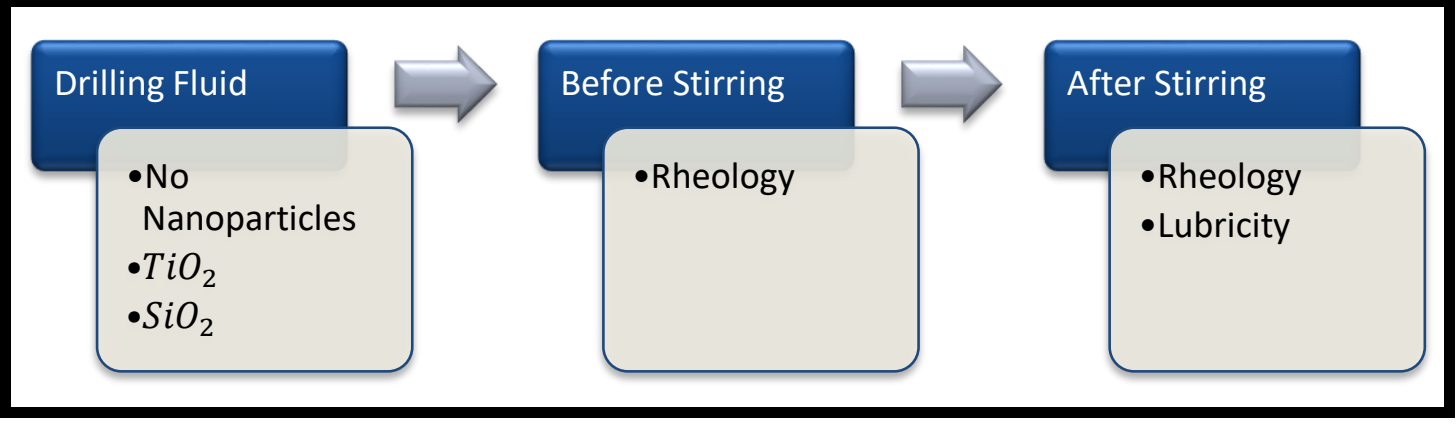

Figure 8: Analysis performed after aging

\section{RESULTS}

After preparing the mud samples and following the procedure described above, extensive results were derived for density and rheology characteristics.

\subsection{Density Results}

Below Table 2 presents the results obtained for the densities of all 5 mud samples in different units, as well as the temperature and $\mathrm{pH}$ of the samples.

Table 2: Densities of mud samples

\begin{tabular}{|c|c|c|c|c|c|c|}
\hline \multicolumn{7}{|c|}{ DENSITY } \\
\hline SAMPLE & PPG & SP GR & $\mathbf{l b} / \mathbf{f t}^{\mathbf{3}}$ & PSI/1000 ft & $\mathbf{T}\left({ }^{\circ} \mathbf{C}\right)$ & PH \\
\hline BASE MUD & 8.83 & 1.06 & 66.14 & 458.98 & 26 & 10 \\
\hline TiO2 0.20 \% & 8.83 & 1.06 & 66.14 & 458.98 & 26 & 10 \\
\hline TiO2 0.60 \% & 8.83 & 1.06 & 66.14 & 458.98 & 26 & 10 \\
\hline SiO2 0.20\% & 8.85 & 1.062 & 66.27 & 459.85 & 26 & 10 \\
\hline SiO2 0.60 \% & 8.90 & 1.068 & 66.64 & 462.44 & 26 & 10 \\
\hline
\end{tabular}




\subsection{Rheology Results}

\subsubsection{Before Aging}

The results of the RPM, viscosity and gel strength rheology obtained for each of the original and 4 nano-particle doped mud samples ( 2 each for titania and silica nano-material) is detailed below, prior to aging. A graph of the shear stress vs the shear rate (RPM) for the different temperatures is also provided to show the trend of the mud samples.

\section{Base Mud:}

Table 3: Gel strength rheology of base mud [cP=centiPoise]

\begin{tabular}{|c|c|c|c|c|c|}
\hline \multicolumn{6}{|c|}{ RHEOLOGY } \\
\hline \multicolumn{6}{|c|}{ BASE MUD } \\
\hline & & \multicolumn{4}{|c|}{ VISCOSITY (cP) } \\
\hline & & \multicolumn{4}{|c|}{ TEMPERATURE $\left({ }^{\circ} \mathrm{C}\right)$} \\
\hline & TEMP & $26^{\circ} \mathrm{C}$ & $40^{\circ} \mathrm{C}$ & $60{ }^{\circ} \mathrm{C}$ & $80^{\circ} \mathrm{C}$ \\
\hline \multirow{7}{*}{ RPM (rad/s) } & 600 & 46.5 & 43 & 43.5 & 42 \\
\hline & 300 & 33 & 31 & 31 & 30 \\
\hline & 200 & 27 & 26 & 26 & 24.5 \\
\hline & 100 & 20.5 & 20 & 19 & 17.5 \\
\hline & 60 & 17 & 17 & 15.5 & 14.5 \\
\hline & 30 & 14 & 13.5 & 13 & 11.5 \\
\hline & 6 & 10 & 10 & 8.5 & 7.5 \\
\hline \multirow{2}{*}{$\begin{array}{l}\text { GEL STRENGTH } \\
\quad\left(l b / 100 f t^{2}\right)\end{array}$} & $10 \mathrm{sec}$ & 9.5 & 9 & 7 & 7 \\
\hline & $10 \mathrm{~min}$ & 14 & 14 & 12 & 14.5 \\
\hline
\end{tabular}




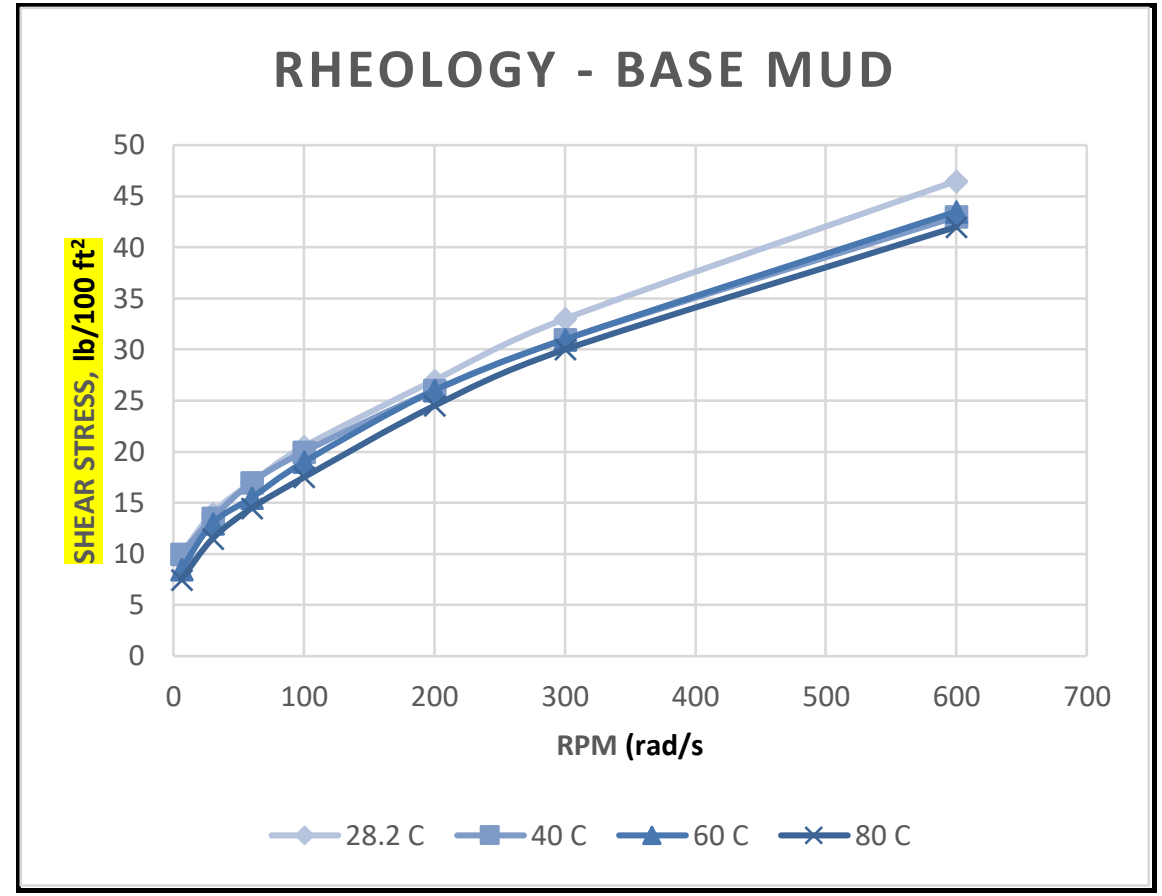

Figure 9: Shear stress rheology of base mud $(\mathrm{C}=$ degrees Celsius $)$

Base Mud with $0.20 \%(w / w) \mathrm{Ti}_{2}$ :

Table 0: Gel strength rheology of base mud with $0.20 \%(\mathrm{w} / \mathrm{w}) \quad \mathrm{Ti}_{2}$ [cP=centiPoise]

\begin{tabular}{|c|c|c|c|c|c|}
\hline \multicolumn{6}{|c|}{$\mathrm{TiO}_{2}(0.20 \% \mathrm{w} / \mathrm{w})$} \\
\hline & & \multicolumn{4}{|c|}{ VISCOSITY (cP) } \\
\hline & & \multicolumn{4}{|c|}{ TEMPERATURE $\left({ }^{\circ} \mathrm{C}\right)$} \\
\hline & & $26^{\circ} \mathrm{C}$ & $40^{\circ} \mathrm{C}$ & $60{ }^{\circ} \mathrm{C}$ & $80^{\circ} \mathrm{C}$ \\
\hline \multirow{7}{*}{ RPM (rad/s) } & 600 & 50 & 49.5 & 47.5 & 51.5 \\
\hline & 300 & 35.5 & 35 & 34 & 37 \\
\hline & 200 & 29.5 & 29.5 & 28.5 & 31 \\
\hline & 100 & 22.5 & 22.5 & 21.5 & 23 \\
\hline & 60 & 19 & 19 & 18.5 & 19 \\
\hline & 30 & 15.5 & 15.5 & 15 & 16 \\
\hline & 6 & 10.5 & 10.5 & 10.5 & 11 \\
\hline \multirow{2}{*}{$\begin{array}{l}\text { GEL STRENGTH } \\
\left(l b / 100 \boldsymbol{f t}^{2}\right)\end{array}$} & $10 \mathrm{sec}$ & 9.5 & 9.5 & 8.5 & 9.5 \\
\hline & $10 \mathrm{~min}$ & 14.5 & 14.5 & 14 & 16.5 \\
\hline
\end{tabular}




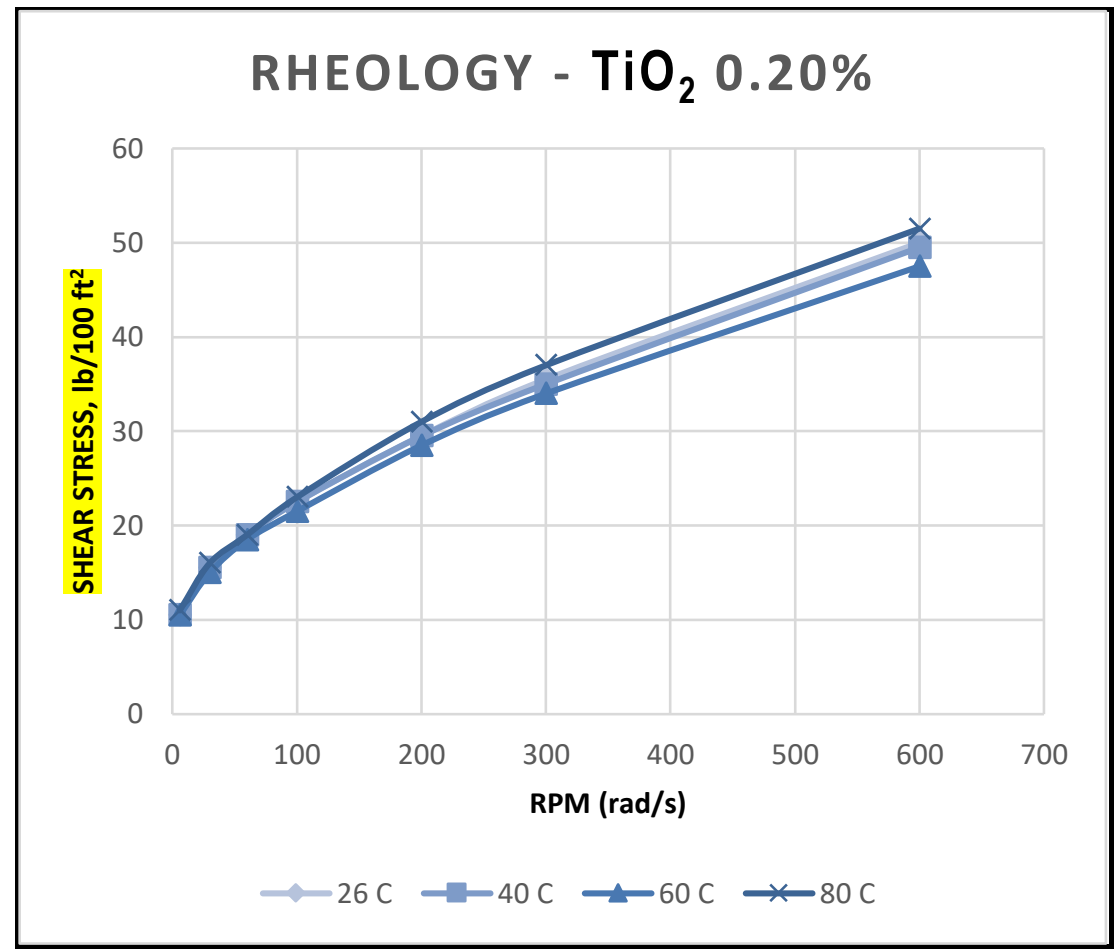

Figure 10: Rheology of base mud with $0.20 \%(w / w) ~ \mathrm{TiO}_{2}(\mathrm{C}=$ degrees Celsius $)$

Base Mud with $0.60 \%(w / w) ~ T i O_{2}$ :

Table 5: Gel strength rheology of base mud with $0.60 \%(w / w) ~ T i O_{2}$ [cP=centiPoise]

\begin{tabular}{|c|c|c|c|c|c|}
\hline \multicolumn{6}{|c|}{ RHEOLOGY } \\
\hline \multicolumn{6}{|c|}{$\mathrm{TiO}_{2}(0.60 \%$ w/w $)$} \\
\hline & & \multicolumn{4}{|c|}{ VISCOSITY (cP) } \\
\hline & & \multicolumn{4}{|c|}{ TEMPERATURE $\left({ }^{\circ} \mathbf{C}\right)$} \\
\hline & & $26^{\circ} \mathrm{C}$ & $40^{\circ} \mathrm{C}$ & $60^{\circ} \mathrm{C}$ & $80^{\circ} \mathrm{C}$ \\
\hline \multirow{7}{*}{$\operatorname{RPM}\left(s^{-1}\right)$} & 600 & 53.5 & 50.5 & 50.5 & 53.5 \\
\hline & 300 & 38 & 37 & 37.5 & 38.5 \\
\hline & 200 & 32.5 & 31.5 & 32 & 33 \\
\hline & 100 & 25 & 24.5 & 25 & 25.5 \\
\hline & 60 & 21 & 21 & 21 & 22 \\
\hline & 30 & 17.5 & 18 & 18 & 18.5 \\
\hline & 6 & 12.5 & 13 & 13 & 14 \\
\hline \multirow{2}{*}{$\begin{array}{l}\text { GEL STRENGTH } \\
\quad\left(l b / 100 f t^{2}\right)\end{array}$} & $10 \mathrm{sec}$ & 12 & 11 & 10 & 11.5 \\
\hline & $10 \mathrm{~min}$ & 16 & 16.5 & 16.5 & 19.5 \\
\hline
\end{tabular}




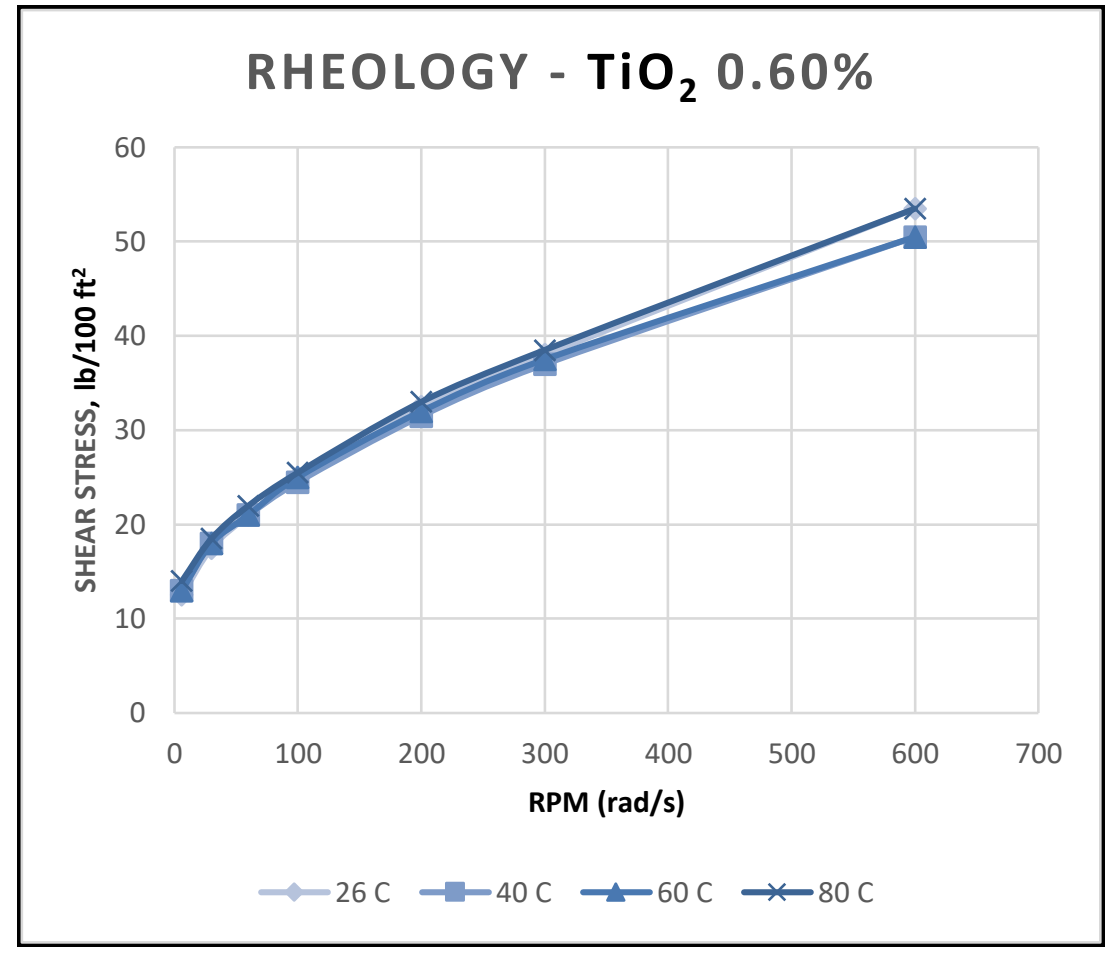

Figure 11: Shear stress rheology of base mud with $0.60 \%(\mathrm{w} / \mathrm{w}) \mathrm{Ti}_{2} \quad(\mathrm{C}=$ degrees Celsius $)$

Base Mud with $0.20 \%(w / w) \mathrm{SiO}_{2}$ :

Table 6: Gel strength rheology of base mud with $0.20 \%$ (w/w) $\mathrm{Si}_{2}$

\begin{tabular}{|c|c|c|c|c|c|}
\hline \multicolumn{6}{|c|}{ RHEOLOGY } \\
\hline \multicolumn{6}{|c|}{$\mathrm{SiO}_{2}(0.20 \% \mathrm{w} / \mathrm{w})$} \\
\hline & & \multicolumn{4}{|c|}{ VISCOSITY (cP) } \\
\hline & & \multicolumn{4}{|c|}{ TEMPERATURE } \\
\hline & & $26^{\circ} \mathrm{C}$ & $40{ }^{\circ} \mathrm{C}$ & $60{ }^{\circ} \mathrm{C}$ & $80^{\circ} \mathrm{C}$ \\
\hline \multirow{7}{*}{ RPM $(\mathrm{rad} / \mathrm{s})$} & 600 & 46 & 41 & 38.5 & 39.5 \\
\hline & 300 & 31.5 & 29.5 & 28 & 27.5 \\
\hline & 200 & 26.5 & 24.5 & 22.5 & 22 \\
\hline & 100 & 19.5 & 18.5 & 16.5 & 15.5 \\
\hline & 60 & 16 & 15.5 & 13 & 12 \\
\hline & 30 & 12.5 & 12 & 10.5 & 9.5 \\
\hline & 6 & 8 & 7.5 & 5.5 & 5.5 \\
\hline \multirow{2}{*}{$\begin{array}{l}\text { GEL STRENGTH } \\
\left(\boldsymbol{l b} / \mathbf{1 0 0} \boldsymbol{f} \boldsymbol{t}^{2}\right)\end{array}$} & $10 \mathrm{sec}$ & 8.5 & 8 & 6 & 6 \\
\hline & $10 \mathrm{~min}$ & 11.5 & 10.5 & 8.5 & 8 \\
\hline
\end{tabular}




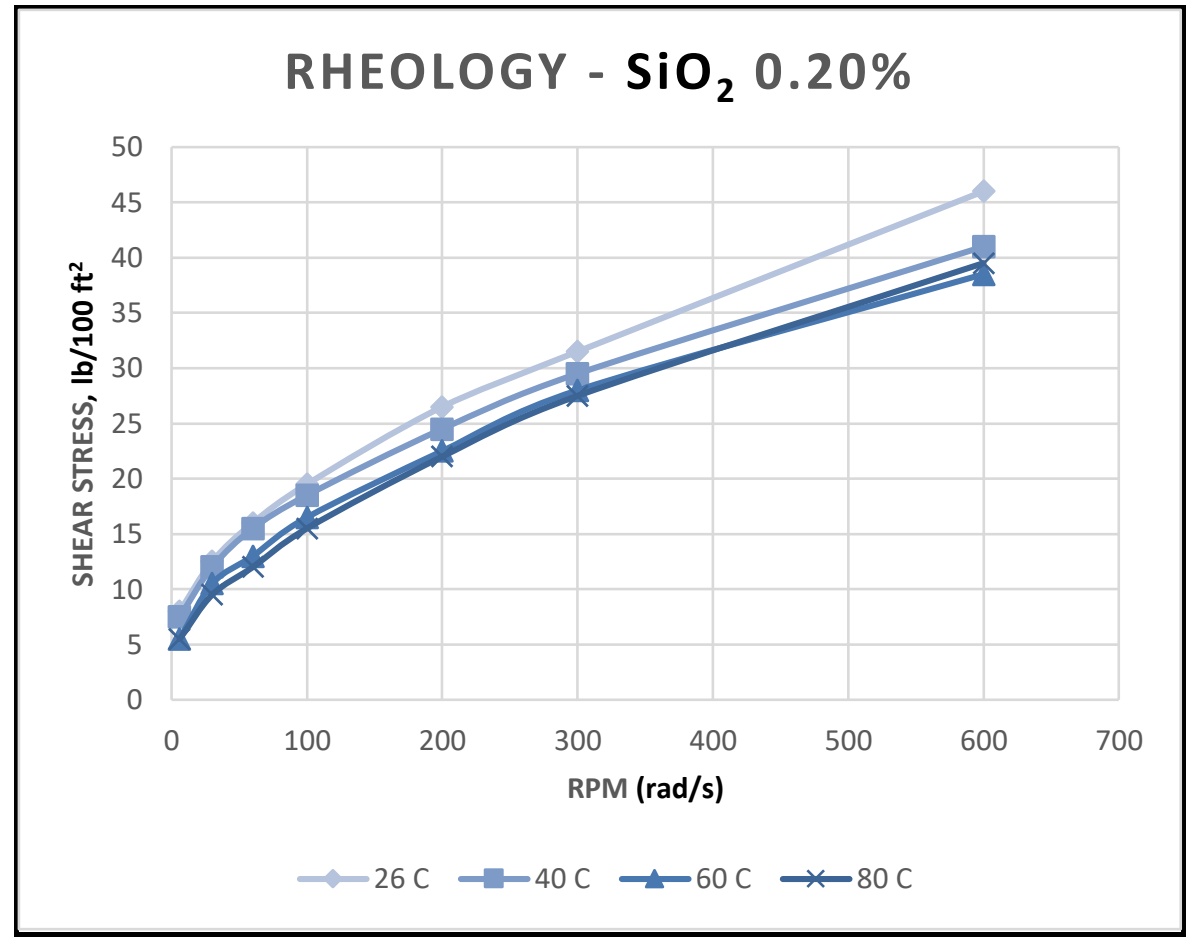

Figure 12: Rheology of base mud with $0.20 \%(\mathrm{w} / \mathrm{w}) \mathrm{SiO}_{2}(\mathrm{C}=$ degrees Celsius $)$

Base Mud with $0.60 \%(w / w)$ Si $\mathrm{O}_{2}$ :

Table 7: Rheology of base mud with $0.60 \%(w / w) \quad S i O_{2}$

\begin{tabular}{|c|c|c|c|c|c|}
\hline \multicolumn{6}{|c|}{ RHEOLOGY } \\
\hline \multicolumn{6}{|c|}{$\mathrm{SiO}_{2}(0.60 \% \mathrm{w} / \mathrm{w})$} \\
\hline & & \multicolumn{4}{|c|}{ VISCOSITY (cP) } \\
\hline & & \multicolumn{4}{|c|}{ TEMPERATURE } \\
\hline & & $26^{\circ} \mathrm{C}$ & $40{ }^{\circ} \mathrm{C}$ & $60{ }^{\circ} \mathrm{C}$ & $80^{\circ} \mathrm{C}$ \\
\hline \multirow{7}{*}{ RPM ( $\mathrm{rad} / \mathrm{s})$} & 600 & 49 & 43.5 & 41 & 41 \\
\hline & 300 & 33.5 & 30.5 & 28.5 & 27 \\
\hline & 200 & 27 & 25.5 & 23.5 & 27.5 \\
\hline & 100 & 19.5 & 19 & 17.5 & 15.5 \\
\hline & 60 & 16 & 15.5 & 14.5 & 12 \\
\hline & 30 & 13 & 12.5 & 10.5 & 9 \\
\hline & 6 & 8.5 & 8 & 6 & 5 \\
\hline \multirow{2}{*}{$\begin{array}{l}\text { GEL STRENGTH } \\
\left(l b / 100 f t^{2}\right)\end{array}$} & $10 \mathrm{sec}$ & 9 & 8 & 7 & 6 \\
\hline & $10 \mathrm{~min}$ & 11 & 10 & 9 & 7.5 \\
\hline
\end{tabular}




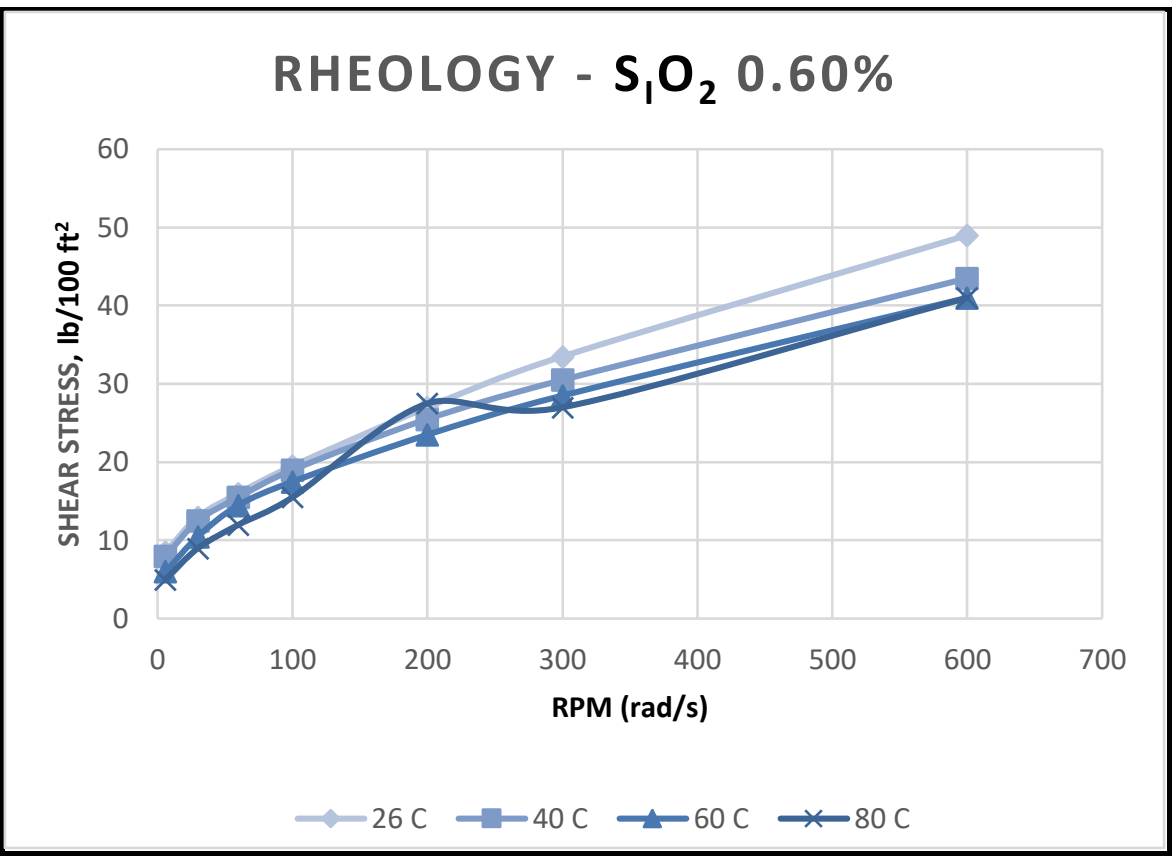

Figure 13: Rheology of base mud with $0.60 \%(w / w) \mathrm{SiO}_{2}(\mathrm{C}=$ degrees Celsius $)$

\subsubsection{After Aging}

After aging the mud samples for 16 hours at $80{ }^{\circ} \mathrm{C}$, the samples were cooled down to $26{ }^{\circ} \mathrm{C}$. Following this the rheological experiments were re-conducted again at one temperature before and after stirring.

\subsection{2-1 Before Stirring}

Table 8: Rheology of aged mud samples before stirring

\begin{tabular}{|c|c|c|c|c|c|c|}
\hline \multicolumn{7}{|c|}{ RHEOLOGY } \\
\hline \multicolumn{7}{|c|}{ AFTER AGING - BEFORE STIRRING } \\
\hline & & \multicolumn{5}{|c|}{ VISCOSITY (cP) } \\
\hline & & \multicolumn{5}{|c|}{ MUD SAMPLE } \\
\hline & & $\begin{array}{l}\text { BASE } \\
\text { MUD }\end{array}$ & $\begin{array}{c}\mathrm{TiO}_{2} \\
0.20 \%\end{array}$ & $\begin{array}{c}\mathrm{TiO}_{2} \\
0.60 \%\end{array}$ & $\begin{array}{c}\mathrm{SiO}_{2} \\
0.20 \%\end{array}$ & $\begin{array}{c}\mathrm{SiO}_{2} \\
0.60 \%\end{array}$ \\
\hline \multirow{7}{*}{ RPM ( rad/s) } & 600 & 39 & 50 & 44 & 54 & 63.5 \\
\hline & 300 & 26.5 & 35 & 30.5 & 38 & 44.5 \\
\hline & 200 & 21 & 28 & 24.5 & 30.5 & 35.5 \\
\hline & 100 & 14 & 20 & 17.5 & 21 & 25.5 \\
\hline & 60 & 11 & 16 & 14 & 17.5 & 19.5 \\
\hline & 30 & 8.5 & 12 & 10.5 & 12.5 & 14.5 \\
\hline & 6 & 5 & 7 & 7 & 6.5 & 8 \\
\hline
\end{tabular}




\begin{tabular}{|c|c|c|c|c|c|c|}
\hline $\begin{array}{c}\text { GEL } \\
\text { STRENGTH } \\
\left(\boldsymbol{l b} / \mathbf{1 0 0} \boldsymbol{f t}^{\mathbf{2}}\right)\end{array}$ & $\mathbf{1 0 ~ s e c}$ & 5 & 6.5 & 7 & 6 & 8 \\
\cline { 2 - 7 } & $\mathbf{1 0} \mathbf{m i n}$ & 7 & 9.5 & 9 & 7.5 & 9 \\
\hline
\end{tabular}

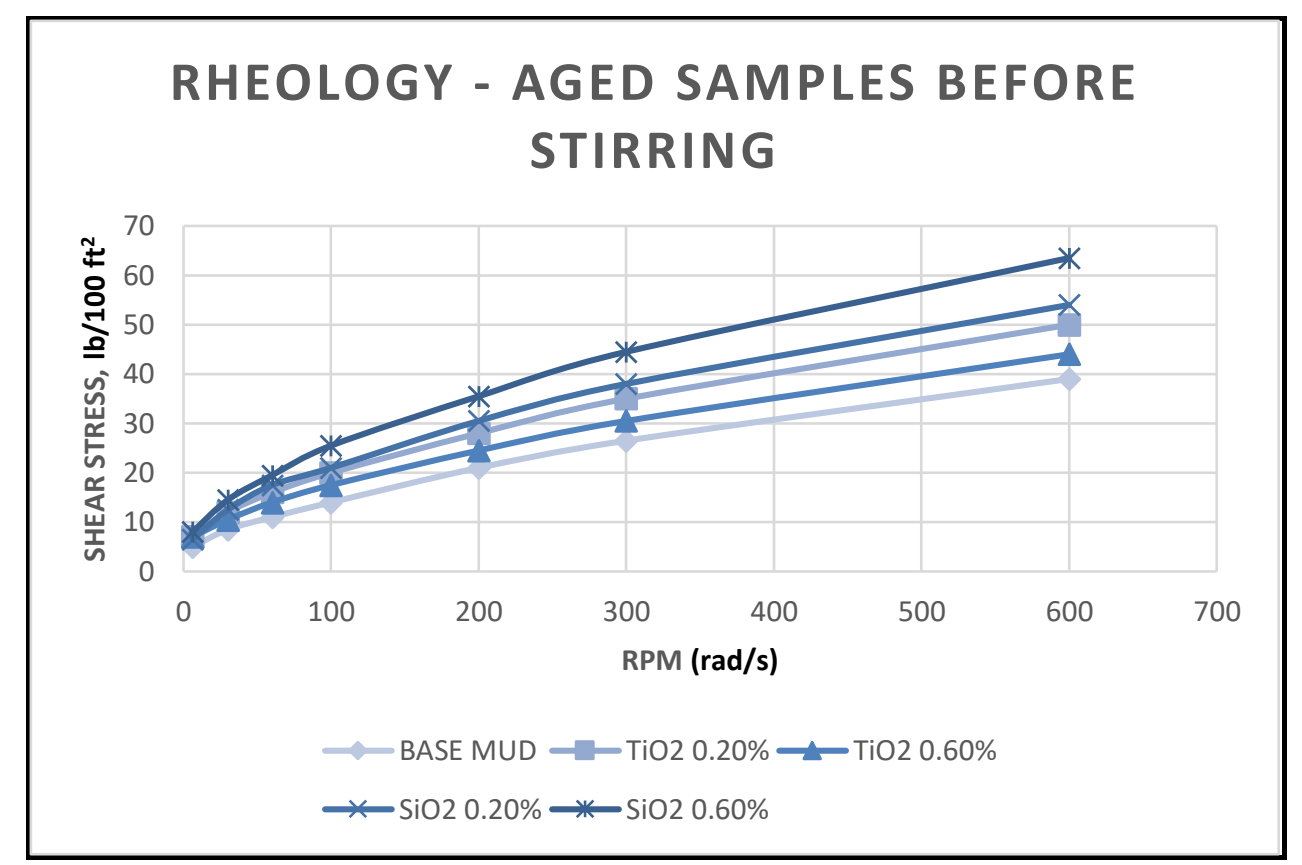

Figure 14: Rheology of aged mud samples before stirring

\subsection{2-2 After Stirring}

Table 9: Rheology of aged mud samples after stirring

\begin{tabular}{|c|c|c|c|c|c|c|}
\hline \multicolumn{7}{|c|}{ RHEOLOGY } \\
\hline \multicolumn{7}{|c|}{ AFTER AGING - AFTER STIRRING } \\
\hline & & \multicolumn{5}{|c|}{ VISCOSITY (cP) } \\
\hline & & \multicolumn{5}{|c|}{ MUD SAMPLE } \\
\hline & & $\begin{array}{l}\text { BASE } \\
\text { MUD }\end{array}$ & $\begin{array}{c}\mathrm{TiO}_{2} \\
\mathbf{0 . 2 0 \%}\end{array}$ & $\begin{array}{c}\mathrm{TiO}_{2} \\
0.60 \%\end{array}$ & $\begin{array}{c}\mathrm{SiO}_{2} \\
0.20 \%\end{array}$ & $\begin{array}{c}\mathrm{SiO}_{2} \\
0.60 \%\end{array}$ \\
\hline \multirow{7}{*}{ RPM $(\mathrm{rad} / \mathrm{s})$} & 600 & 26.5 & 34.5 & 32 & 33 & 38.5 \\
\hline & 300 & 18 & 24 & 22 & 22 & 26 \\
\hline & 200 & 15 & 19.5 & 18 & 18.5 & 20.5 \\
\hline & 100 & 10.5 & 13.5 & 13 & 12 & 14.5 \\
\hline & 60 & 7.5 & 11 & 10 & 8.5 & 11 \\
\hline & 30 & 5.5 & 8 & 7.5 & 6 & 8 \\
\hline & 6 & 3 & 4.5 & 4 & 3.5 & 4.5 \\
\hline & $10 \mathrm{sec}$ & 4 & 4.5 & 5 & 4.5 & 5 \\
\hline
\end{tabular}




\begin{tabular}{|c|c|c|c|c|c|c|}
\hline $\begin{array}{c}\text { GEL } \\
\text { STRENGTH } \\
\left(\boldsymbol{l b} / \mathbf{1 0 0} \boldsymbol{f t}^{2}\right)\end{array}$ & $\mathbf{1 0} \mathbf{~ m i n}$ & 7 & 7 & 8 & 6 & 7 \\
\hline
\end{tabular}

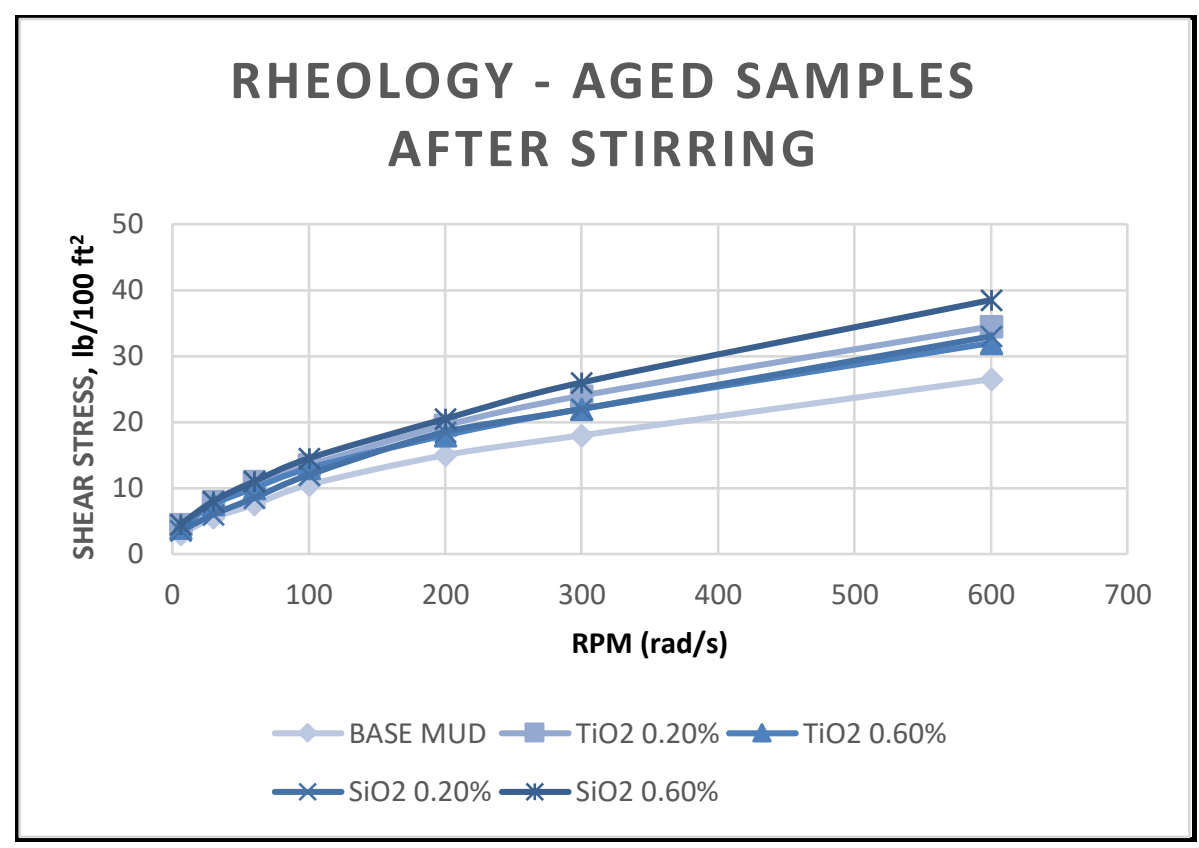

Figure 15: Rheology of aged mud samples after stirring

\subsection{Lubricity Results}

Before evaluating the mud samples, the correction factor for the lubricity ring and lubricity block was calculated using equation (5). The result obtained is shown below:

Correction Factor $=\frac{34}{36}=0.94$

\subsubsection{Before Aging}

Similar to the rheology tests, the lubricity of the original and 4 nano-doped mud samples were evaluated at different temperatures as well. In this case, the data extracted is the torque reading. The following tables and graphs show the torque readings of the mud samples at different temperatures. The analysis of the lubricity is then subsequently conducted.

\section{Base Mud:}

Table 10: Torque readings of base mud

\begin{tabular}{|c|c|c|c|c|c|}
\hline \multicolumn{7}{|c|}{ LUBRICITY TEST } \\
\hline \multicolumn{5}{|c|}{ BASE MUD } \\
\hline & & \multicolumn{4}{c|}{ TEMPERATURE } \\
\hline & TIME (min) & $\mathbf{2 6}^{\circ} \mathbf{C}$ & $\mathbf{4 0}^{\circ} \mathbf{C}$ & $\mathbf{6 0}^{\circ} \mathbf{C}$ & $\mathbf{8 0}^{\circ} \mathbf{C}$ \\
\hline $\begin{array}{c}\text { TORQUE } \\
\text { READING }\end{array}$ & $\mathbf{1}$ & 37.5 & 42.3 & 45.2 & 43.6 \\
\cline { 2 - 6 } & $\mathbf{2}$ & 39.5 & 42.4 & 44.4 & 44.4 \\
\hline
\end{tabular}




\begin{tabular}{|c|c|c|c|c|c|}
\hline (inch-pounds) & $\mathbf{3}$ & 40 & 42 & 44.4 & 45.5 \\
\cline { 2 - 6 } & $\mathbf{4}$ & 40 & 41.4 & 44.6 & 45.8 \\
\cline { 2 - 6 } & $\mathbf{5}$ & 39.9 & 40.7 & 44.6 & 46.1 \\
\cline { 2 - 6 } & $\mathbf{6}$ & 39.6 & 41.1 & 45 & 46.6 \\
\hline
\end{tabular}

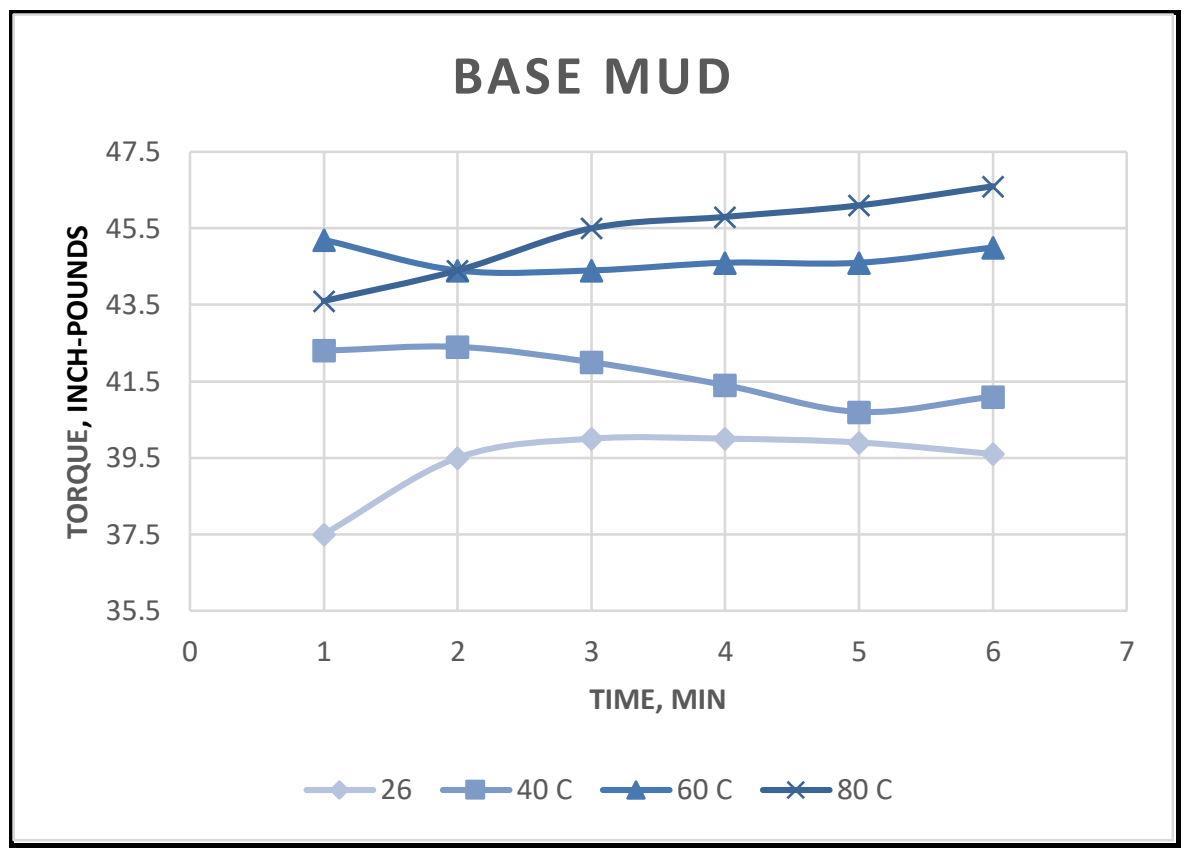

Figure 16: Torque readings of base mud $(\mathrm{C}=$ degrees Celsius $)$

Base Mud with $0.20 \%(w / w) \mathrm{Ti}_{2}$ :

Table 11: Torque readings of base mud with $0.20 \%(\mathrm{w} / \mathrm{w}) \mathrm{Ti} \mathrm{O}_{2}$

\begin{tabular}{|c|c|c|c|c|c|}
\hline \multicolumn{5}{|c|}{ LUBRICITY TEST } \\
\hline & $\mathrm{TiO}_{2}(\mathbf{0 . 2 0} \%$ w/w) \\
\hline & TIME (min) & \multicolumn{4}{c|}{ TEMPERATURE } \\
\hline & $\mathbf{1}$ & 42.4 & 42.7 & 47.9 & 44.4 \\
\hline \multirow{4}{*}{$\begin{array}{c}\text { TORQUE } \\
\text { READING (inch- } \\
\text { pounds) }\end{array}$} & $\mathbf{2}$ & 44.5 & 43.3 & 48.5 & 44.6 \\
\cline { 2 - 6 } & $\mathbf{3}$ & 44.2 & 44.1 & 48.6 & 44.4 \\
\cline { 2 - 6 } & $\mathbf{4}$ & 44.6 & 45.2 & 48.4 & 46.3 \\
\cline { 2 - 6 } & $\mathbf{5}$ & 45.9 & 46 & 47.7 & 45.7 \\
\cline { 2 - 6 } & $\mathbf{6}$ & 46.1 & 45.3 & 47 & 45.6 \\
\hline
\end{tabular}




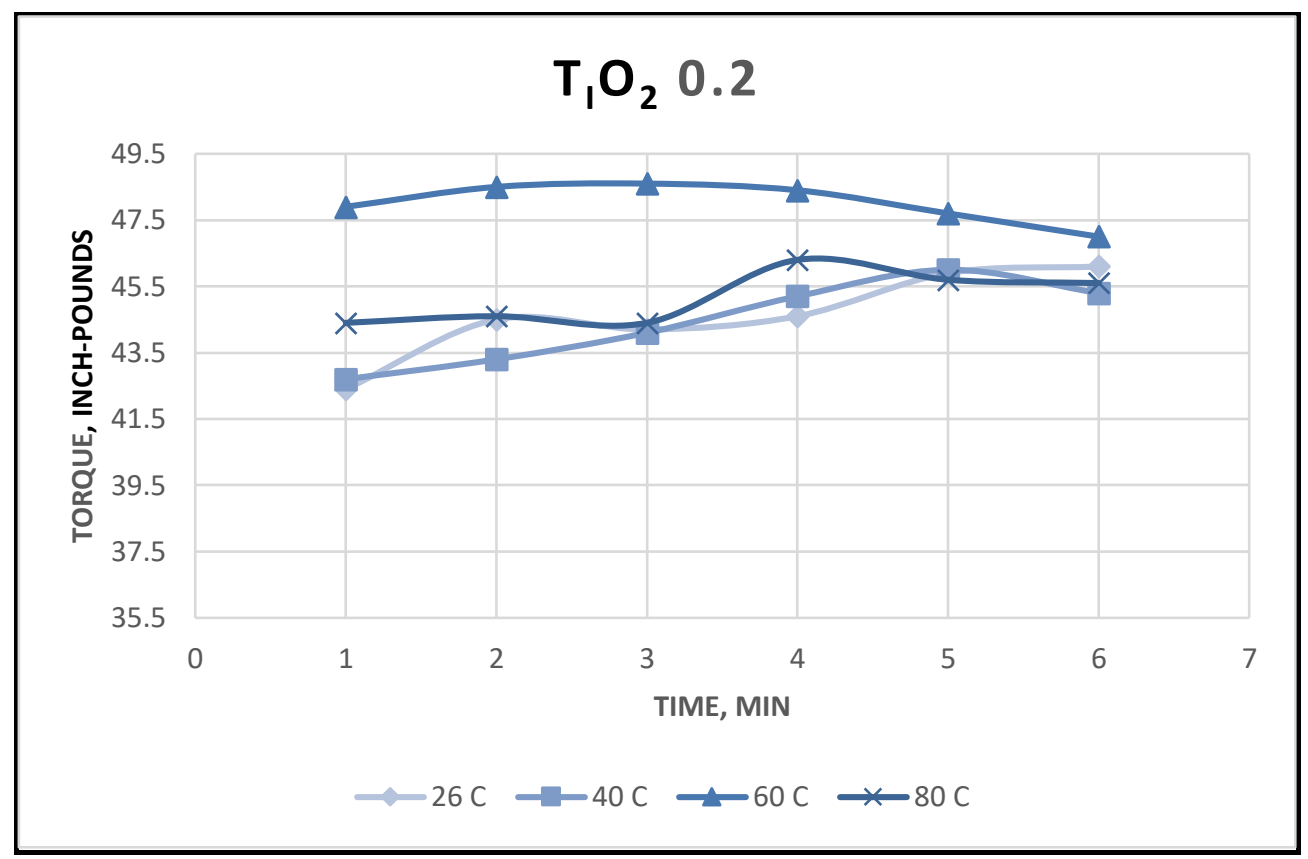

Figure 17: Torque readings of base mud with $0.20 \%(\mathrm{w} / \mathrm{w}) \mathrm{Ti}_{2}(\mathrm{C}=$ degrees Celsius)

Base Mud with 0.60\% (w/w) $\mathrm{Ti}_{2}$ :

Table 12: Torque readings of base mud with $0.60 \%(\mathrm{w} / \mathrm{w}) \mathrm{Ti} \mathrm{O}_{2}$

\begin{tabular}{|c|c|c|c|c|c|}
\hline \multicolumn{6}{|c|}{ LUBRICITY TEST } \\
\hline \multicolumn{6}{|c|}{$\mathrm{TiO}_{2}(0.60 \%$ w/w $)$} \\
\hline & & \multicolumn{4}{|c|}{ TEMPERATURE } \\
\hline & TIME (min) & $26^{\circ} \mathrm{C}$ & $40^{\circ} \mathrm{C}$ & $60^{\circ} \mathrm{C}$ & $80{ }^{\circ} \mathrm{C}$ \\
\hline \multirow{6}{*}{$\begin{array}{c}\text { TORQUE } \\
\text { READING (inch- } \\
\text { pounds) }\end{array}$} & 1 & 40.2 & 39.2 & 39.3 & 39 \\
\hline & 2 & 40 & 37.7 & 38.4 & 39.4 \\
\hline & 3 & 40 & 37.4 & 37.9 & 39.3 \\
\hline & 4 & 39.3 & 36.7 & 37.6 & 39.4 \\
\hline & 5 & 38.7 & 35.9 & 37.6 & 39 \\
\hline & 6 & 37.9 & 36.2 & 37.4 & 39.6 \\
\hline
\end{tabular}




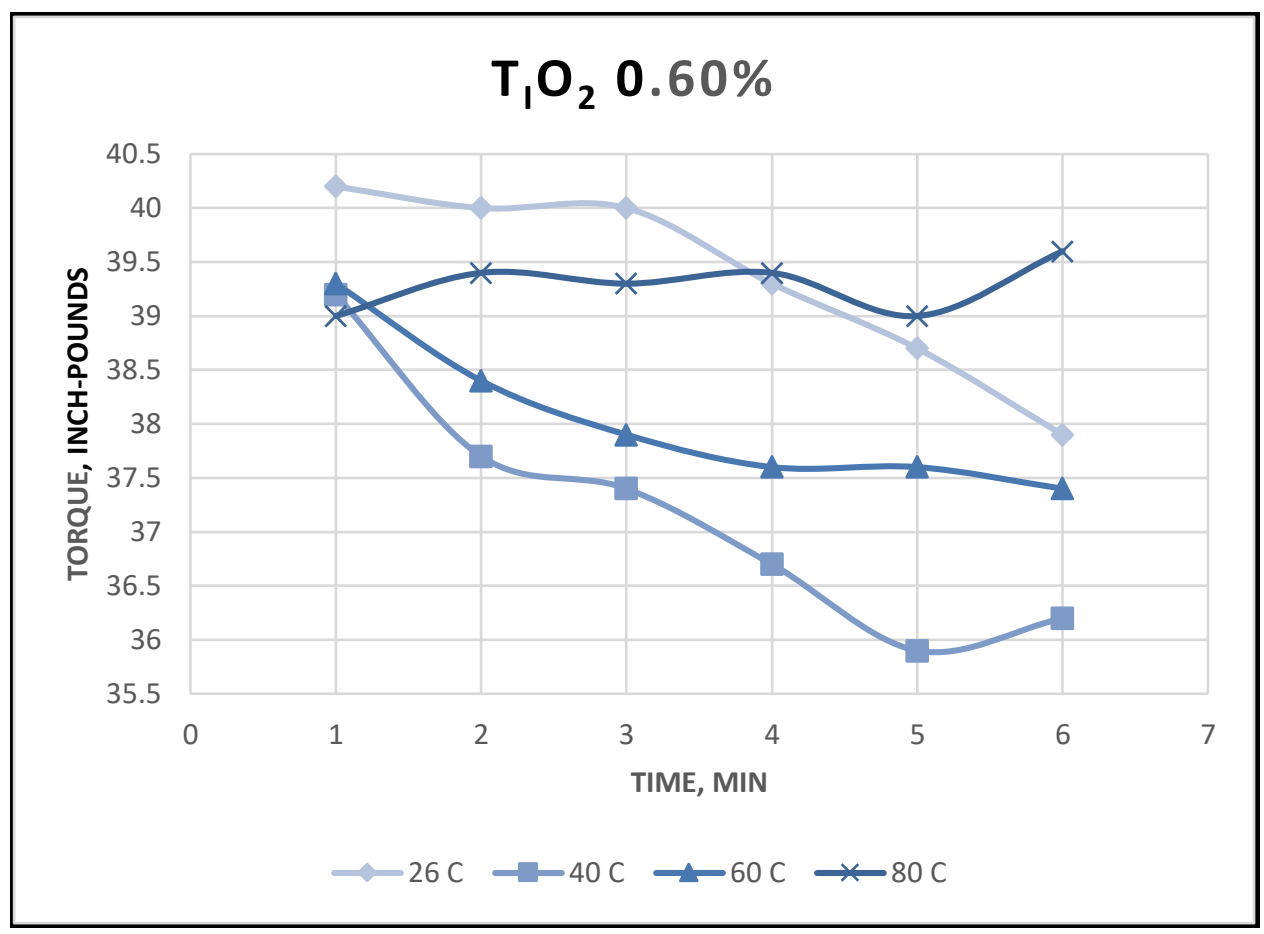

Figure 18: Torque readings of base mud with $0.60 \%(w / w) ~ \mathrm{TiO}_{2}(\mathrm{C}=$ degrees Celsius $)$

Base Mud with $0.20 \%(w / w)$ Si $O_{2}$

Table 13: Torque readings of base mud with $0.20 \%(\mathrm{w} / \mathrm{w}) \mathrm{Si} \mathrm{O}_{2}$

\begin{tabular}{|c|c|c|c|c|c|}
\hline \multicolumn{6}{|c|}{ LUBRICITY TEST } \\
\hline \multicolumn{6}{|c|}{$\mathrm{SiO}_{2}(0.20 \%$ w/w $)$} \\
\hline & & \multicolumn{4}{|c|}{ TEMPERATURE } \\
\hline & TIME (min) & $26^{\circ} \mathrm{C}$ & $40{ }^{\circ} \mathrm{C}$ & $60^{\circ} \mathrm{C}$ & $80{ }^{\circ} \mathrm{C}$ \\
\hline \multirow{6}{*}{$\begin{array}{c}\text { TORQUE } \\
\text { READING (inch- } \\
\text { pounds) }\end{array}$} & 1 & 37 & 40.6 & 42.5 & 43.9 \\
\hline & 2 & 38.8 & 41.2 & 42.7 & 44.5 \\
\hline & 3 & 39.1 & 42.3 & 43.3 & 44.1 \\
\hline & 4 & 39.8 & 42.5 & 43.9 & 44.7 \\
\hline & 5 & 40.7 & 42.8 & 43.6 & 44.7 \\
\hline & 6 & 40.2 & 43.1 & 43.4 & 44.2 \\
\hline
\end{tabular}




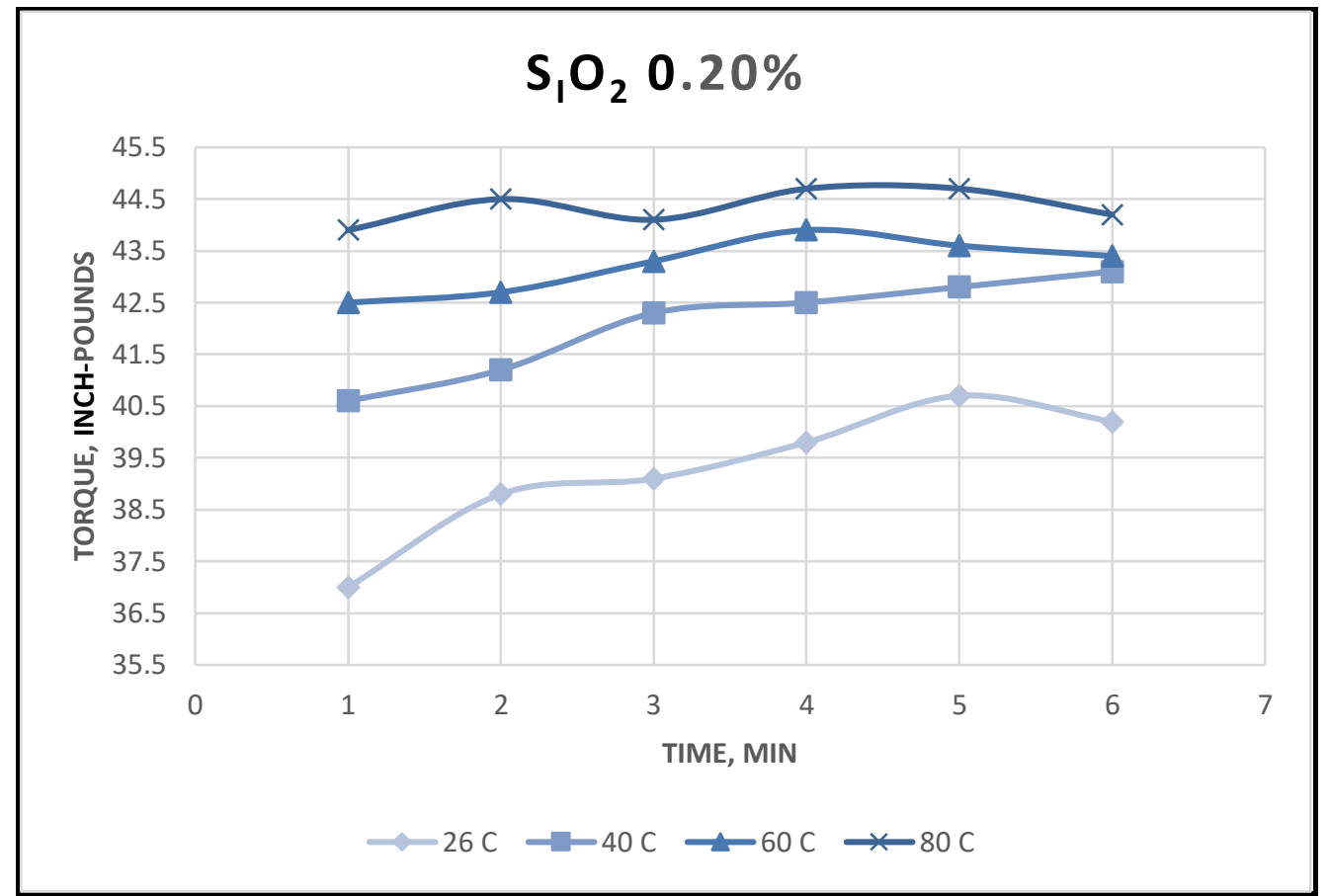

Figure 19: Torque readings of base mud with $0.20 \%(\mathrm{w} / \mathrm{w}) \mathrm{SiO}_{2}(\mathrm{C}=$ degrees Celsius $)$

Base Mud with $0.60 \%(w / w)$ Si $O_{2}$ :

Table 14: Torque readings of base mud with $0.60 \%$ (w/w) $\mathrm{Si}_{2}$

\begin{tabular}{|c|c|c|c|c|c|}
\hline \multicolumn{6}{|c|}{ LUBRICITY TEST } \\
\hline \multicolumn{6}{|c|}{$\mathrm{SiO}_{2}(0.60 \%$ w/w $)$} \\
\hline & & \multicolumn{4}{|c|}{ TEMPERATURE } \\
\hline & TIME (min) & $26^{\circ} \mathrm{C}$ & $40^{\circ} \mathrm{C}$ & $60{ }^{\circ} \mathrm{C}$ & $80{ }^{\circ} \mathrm{C}$ \\
\hline \multirow{6}{*}{$\begin{array}{c}\text { TORQUE } \\
\text { READING (inch- } \\
\text { pounds) }\end{array}$} & 1 & 47 & 43.2 & 43.9 & 44.8 \\
\hline & 2 & 46.2 & 41.6 & 44.7 & 44.4 \\
\hline & 3 & 45.2 & 41 & 44 & 43.7 \\
\hline & 4 & 43.8 & 40.6 & 43.7 & 43.5 \\
\hline & 5 & 42.6 & 40 & 43.2 & 43.1 \\
\hline & 6 & 43.4 & 40.4 & 43.1 & 42.8 \\
\hline
\end{tabular}




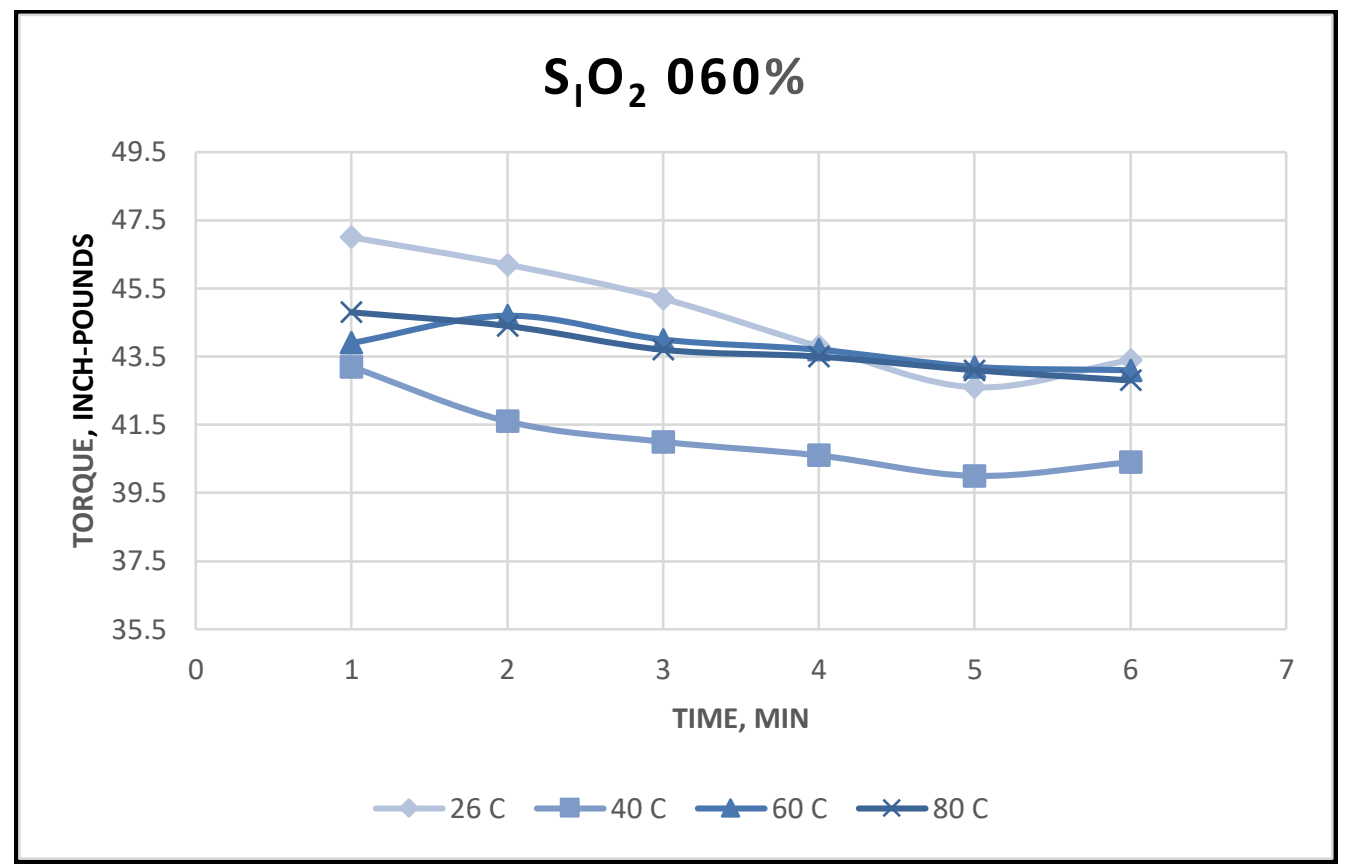

Figure 20: Torque readings of base mud with $0.60 \%(\mathrm{w} / \mathrm{w}) \mathrm{SiO}_{2}(\mathrm{C}=$ degrees Celsius $)$

\subsubsection{After Aging}

The lubricity test was repeated for all 5 mud samples after the aging process and stirring them in the mixer for 10 minutes. The results are shown below.

Table 15: Torque readings of aged mud samples after stirring

\begin{tabular}{|c|c|c|c|c|c|c|}
\hline \multicolumn{7}{|c|}{ LUBRICITY TEST } \\
\hline \multicolumn{7}{|c|}{ AFTER AGING - AFTER STIRRING } \\
\hline & & \multicolumn{5}{|c|}{ MUD SAMPLES } \\
\hline & $\begin{array}{l}\text { TIME } \\
\text { (min) }\end{array}$ & $\begin{array}{l}\text { BASE } \\
\text { MUD }\end{array}$ & $\begin{array}{c}\mathrm{TiO}_{2} \\
0.20 \%\end{array}$ & $\begin{array}{c}\mathrm{TiO}_{2} \\
0.60 \%\end{array}$ & $\begin{array}{c}\mathrm{SiO}_{2} \\
0.20 \%\end{array}$ & $\begin{array}{c}\mathrm{SiO}_{2} \\
0.60 \%\end{array}$ \\
\hline \multirow{6}{*}{$\begin{array}{l}\text { TORQUE } \\
\text { READING }\end{array}$} & 1 & 28 & 36.5 & 33.1 & 40.4 & 37.5 \\
\hline & 2 & 32.9 & 35.8 & 33.6 & 38.8 & 38.4 \\
\hline & 3 & 34.1 & 36.8 & 33.6 & 37.8 & 38 \\
\hline & 4 & 34.8 & 36.1 & 33.8 & 37.1 & 38.4 \\
\hline & 5 & 34.3 & 36 & 33.7 & 37.3 & 38.4 \\
\hline & 6 & 34.1 & 35.9 & 33.9 & 36.9 & 38 \\
\hline
\end{tabular}




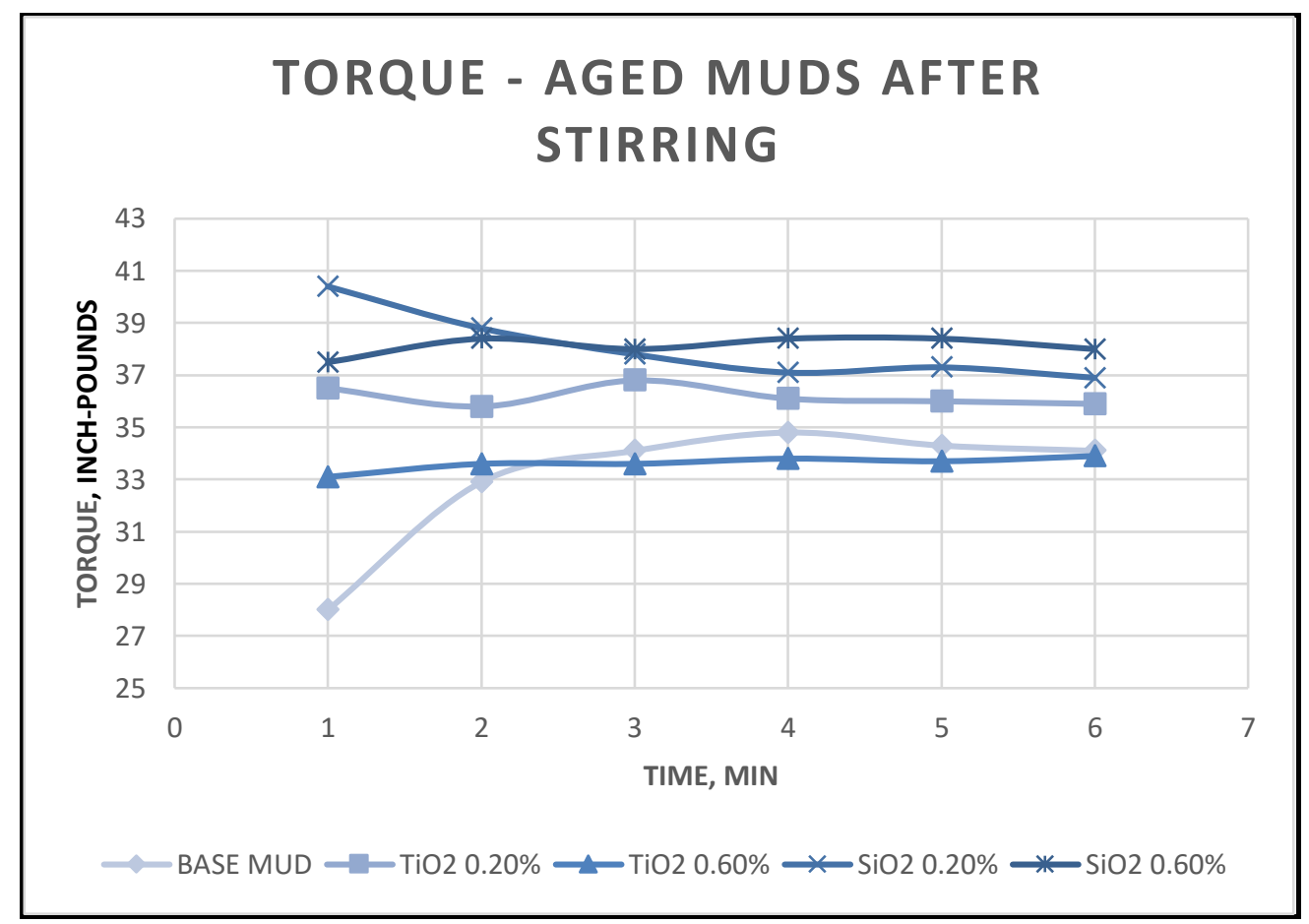

Figure 21: Torque readings of aged mud samples after stirring

\section{ANALYSIS AND DISCUSSION}

In this section, we interpret the results presented earlier. Each set of results pertaining to different aspects of the investigation is addressed individually i.e. density, rheology and lubricity. The density unit used is that consistent with modern petroleum engineering, namely lbm/gal (also known as ppg i.e. pounds per gallon).

\subsection{Density}

Inspection of table 2 reveals that density of the mud samples was very similar after the addition of nanoparticles. There was only a small increase when silica nanoparticles were added, while the variation in concentration of titania nanoparticles did not show any tangible alteration. This increment observed with silica nanoparticles was proportional to the concentration of these nano-particles in the base mud, and this has been calculated and listed in Table 16. These results suggest that silica nanoparticles could have acted as weighting agents which could be applicable in actual water-based mud suspensions in the field where even a slight modification in density may be beneficial to drilling (Mostafavi et al. 2011).

Table 16: Variation in density with nanoparticles

\begin{tabular}{|c|c|c|}
\hline \multicolumn{3}{|c|}{ DENSITY } \\
\hline SAMPLE & PPG & INCREASE (\%) \\
\hline BASE MUD $=$ & 8.83 & \\
\hline $\mathrm{TiO} 20.20 \%=$ & 8.83 & 0 \\
\hline TiO2 $0.60 \%=$ & 8.83 & 0 \\
\hline $\mathrm{SiO} 20.20 \% \Delta$ & 8.85 & 0.23 \\
\hline SiO2 $0.60 \% \triangle$ & 8.90 & 0.79 \\
\hline
\end{tabular}




\subsection{Rheology-before aging}

In general, the results of the rheology tests (before aging) of the mud samples doped with nano-particles, which are plotted from figures 10-15 demonstrate the normal trend of typical drilling muds i.e. without nano-particles (sample 1, figure 9). The analysis of the rheological properties was done using the Bingham (viscoplastic) model and the curves of each mud sample were adapted to this model. However, this model is not representative of the drilling mud behaviour at low shear rates. The plastic viscosity, yield point and apparent viscosity were calculated using equations (1), (2) and (3), respectively. An example of the calculation of these parameters for the base mud at $26^{\circ} \mathrm{C}$ follows:

\section{Plastic Viscosity:}

$P V_{\text {Base } \operatorname{Mud}\left(2 \mathbf{2 0}^{\circ} \mathrm{c}\right)}=600 R P M$ reading $-300 R P M$ reading $=46.5-33=13.5 \mathrm{cp}$

\section{Yield Point:}

$Y P_{\text {Base } \operatorname{Mud}}\left(2 \mathbf{2 6}^{\circ} \mathrm{c}\right)=300 \mathrm{RPM}$ reading $-P V=33-13.5=19.5 \mathrm{lb} / 100 \mathrm{ft} \mathrm{t}^{2}$

\section{Apparent Viscosity:}

$$
A V_{\text {Base Mud }\left(26^{\circ} \mathrm{c}\right)}=\frac{600 \mathrm{RPM} \text { reading }}{2}=\frac{46.5}{2}=23.25 \mathrm{cp}
$$

The same procedure was repeated for the other results obtained in Tables 3-9.

Table 17: Results of PV, YP, YP/PV and AV of mud samples before aging 


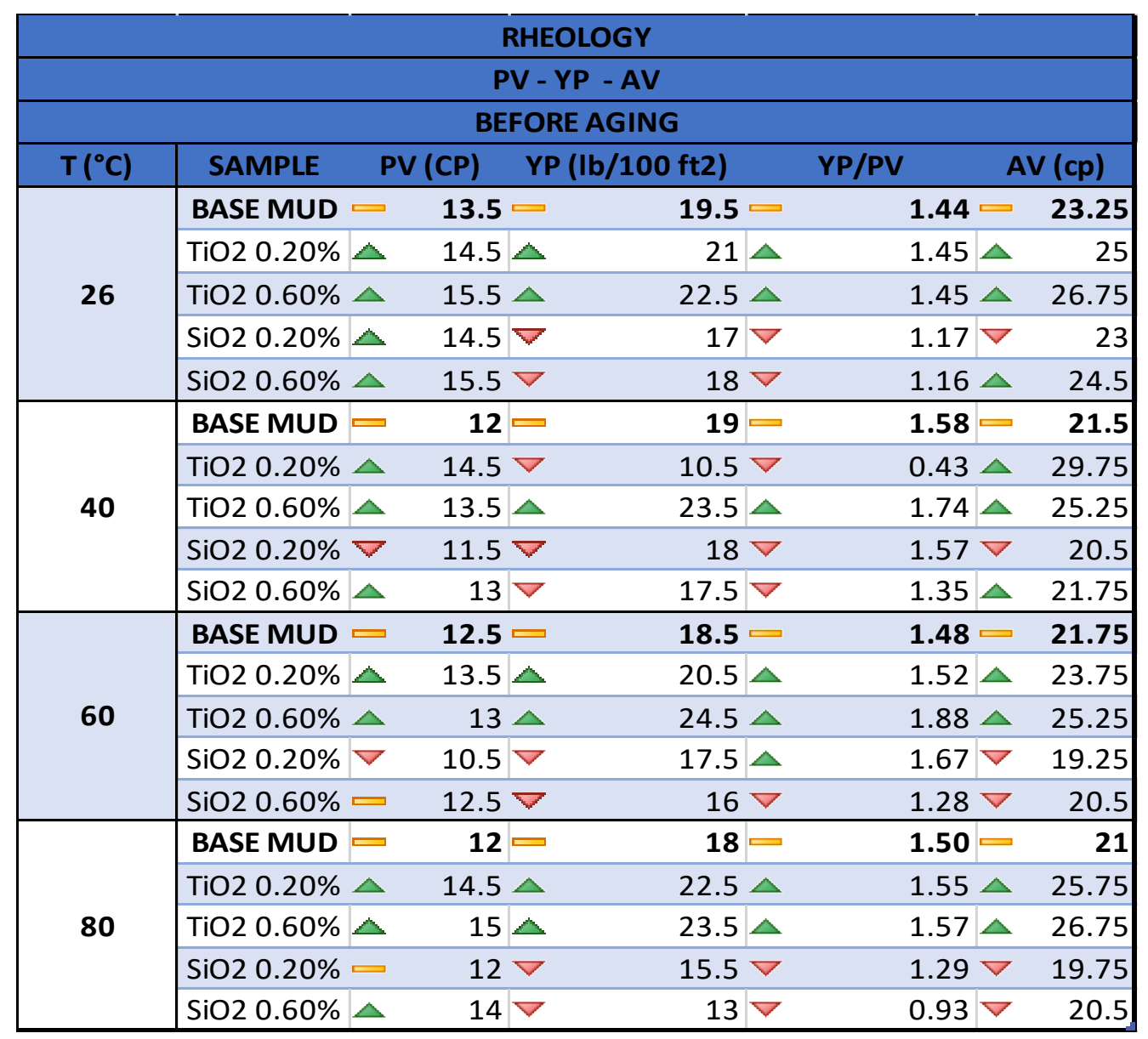

Table 17 summarizes these calculations using the Bingham model (a yield stress is required to initiate flow), and presents the increase (green arrow) and decrease (red arrow) of these parameters compared to the base mud (yellow dash) for each temperature. The evaluation of the rheology at different temperatures showed that the viscosity readings were different for each case, although, they were close to the base mud results. From figures 9-13, it is evident that titania nanoparticles provided higher viscosity than base mud or silica-doped mud. Silica nanoparticles infact reduced the viscosity of the mud. In general, the viscosity of all the mud samples tended to increase at high shear rates. Moreover, in the case of silica nanoparticles and base mud, the viscosity was reduced when the temperature was increased. However, in the case of titania nanoparticles, it is apparent that the variations of viscosity at different shear rates remained almost the same for all the temperatures. A possible explanation for this behaviour is that silica nanoparticles are hydrophilic, so that the amount of solid particles in the base mud is infact drastically reduced and they do not improve therefore the viscosity of the base mud as titania nano-particles. It is also important to note that in general, titania nanoparticles showed an increase in plastic viscosity (PV), yield point (YP) and apparent viscosity (AV), although they exhibit a decrease in yield point when the concentration is lower i.e. $0.20 \%(\mathrm{w} / \mathrm{w})$ at $40{ }^{\circ} \mathrm{C}$. The addition of silica nanoparticles results in a decrease in the yield point, and a similar trend in apparent viscosity although there is a deviation from this at a concentration of $0.60 \%(\mathrm{w} / \mathrm{w})$ where viscosity increases at $26^{\circ} \mathrm{C}$ and $40{ }^{\circ} \mathrm{C}$. Some fluctuation in results is also evident with temperature and a consistent trend is not attained for plastic viscosity with silica. Plastic viscosity tends to increase in drilling fluids due to an excess in the number of particles, which in turn causes an increase in the mechanical friction between solids and liquids in the mud system (Baker Huges 1995). Taking into account this antecedent and the 
concentrations used for each nanoparticle, $0.20 \%(\mathrm{w} / \mathrm{w})$ and $0.60 \%(\mathrm{w} / \mathrm{w})$, and assuming that the morphology of both nanoparticles was spherical the number of titania particles per $350 \mathrm{ml}$ was roughly $1.17 \times 10^{16}$ and $3.51 \times 10^{16}$, respectively. However, the number of silica particles for the same amount of liquid was about $6.33 \times 10^{16}$ and $19.01 \times 10^{16}$ which is significantly larger and may be counter-productive for mud rheology. Furthermore, the calculation is not exact since the structure of both nanoparticles are not exactly spherical, as described in the property tables given in the Appendix, but approximations. A visualization of the variation of plastic viscosity for all five samples is given in Figure. 22

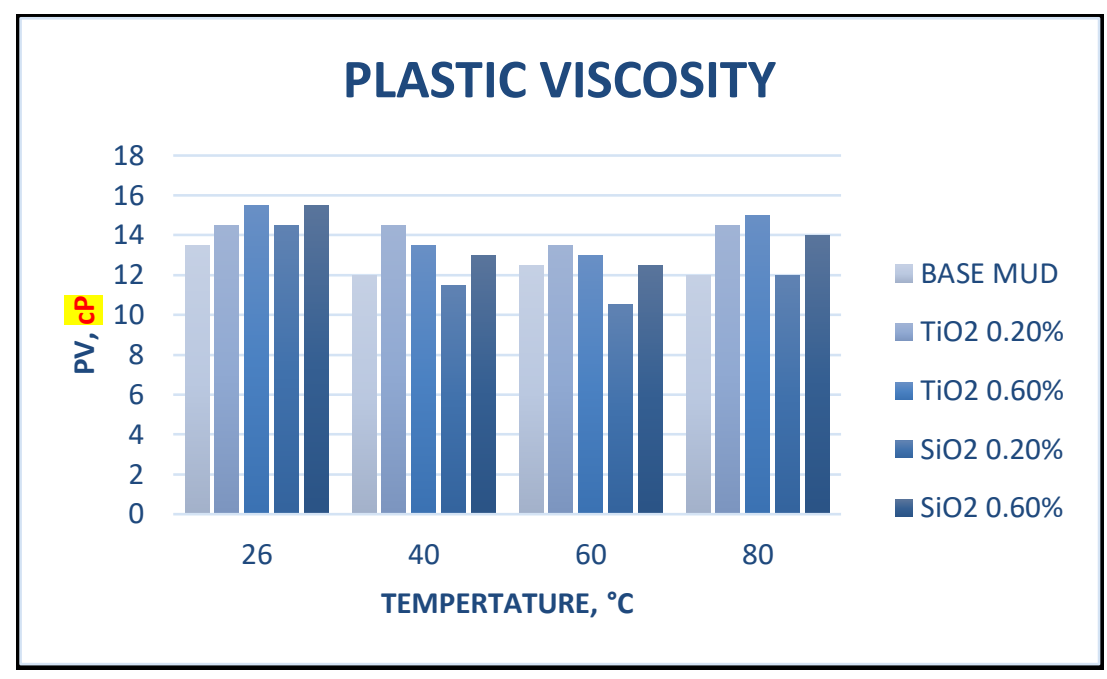

Figure 22: Plastic viscosity before aging

In the case of silica nanoparticles, the super hydrophilic characteristic (due to their coating agent) may have reduced the amount of solid silica nanoparticles dispersed in the drilling mud. The plastic viscosity achieved when titania was added almost invariant with different temperatures and did not follow the normal decreasing trend of the base mud. A normal mud tends to decrease in plastic viscosity when its temperature is raised since the viscosity of the water decreases (Baker Huges, 1995). This effect may furthermore contribute to improved thermal conductivity of titania nanoparticles in the base mud. Regarding the yield point, this parameter is an indicator of the chemical attraction of the particles in the drilling mud. When the balance is broken, the yield point tends to increase. This may arise due to the addition of inert solids and the volume concentration of solids although many other micro-structural features may also contribute. In the case of titania nanoparticles, these nano-particles may not have interacted with the rest of the additives, acting as inert particles, in the drilling mud which may manifest in an increase in yield point. In the case of silica nanoparticles, the super hydrophilic feature could have caused a reduction in yield point since these particles were probably dissolved in the drilling mud, which is water-based which results in a decrease in volume concentration of solids. Therefore, the addition of titania nanoparticles in the base mud indicates that this nano-particle doped mud sample requires greater shear stress to initiate motion of the drilling mud, as shown in figure 23. Conversely the addition of silica nanoparticles requires less stress for mobilizing flow of the drilling mud. Although lower yield stress may have advantages it can also lead to problems in the reduced ability of the drilling mud to suspend drill cuttings when drilling. 


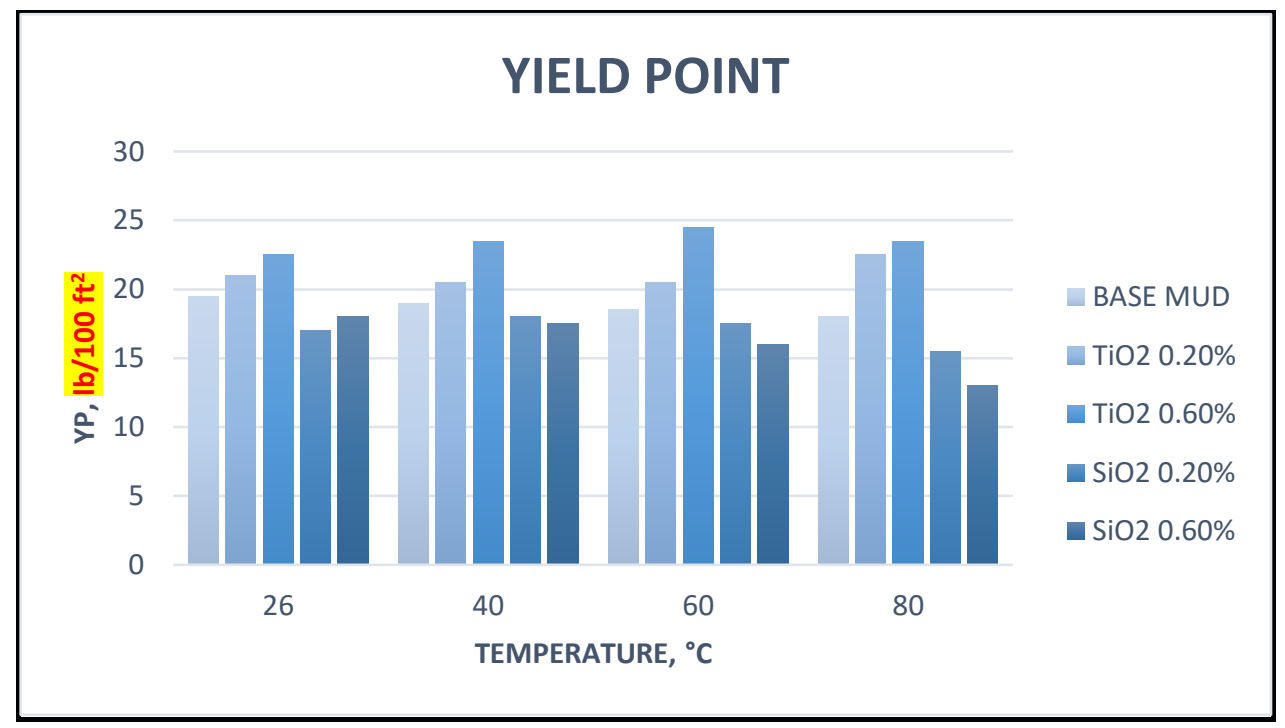

Figure 23: Yield point before aging

The relationship between both parameters (YP/PV), yield point and plastic viscosity, is useful to determine the efficiency of the drilling mud when cleaning the well. A low plastic viscosity and a high yield point give an optimum efficiency. The results in figure $\mathbf{2 4}$ show that titania nanoparticles achieve the highest increase in this relationship, which is proportional to the increase in concentration and is slightly greater than the base mud. On the other hand, silica nanoparticles showed the lowest efficiency and an inversely proportional relationship with the increase in concentration. Therefore, the addition of silica nanoparticles could cause problems related to the cleaning efficiency of the drilling mud, whereas titania nanoparticles could contribute to improving it.

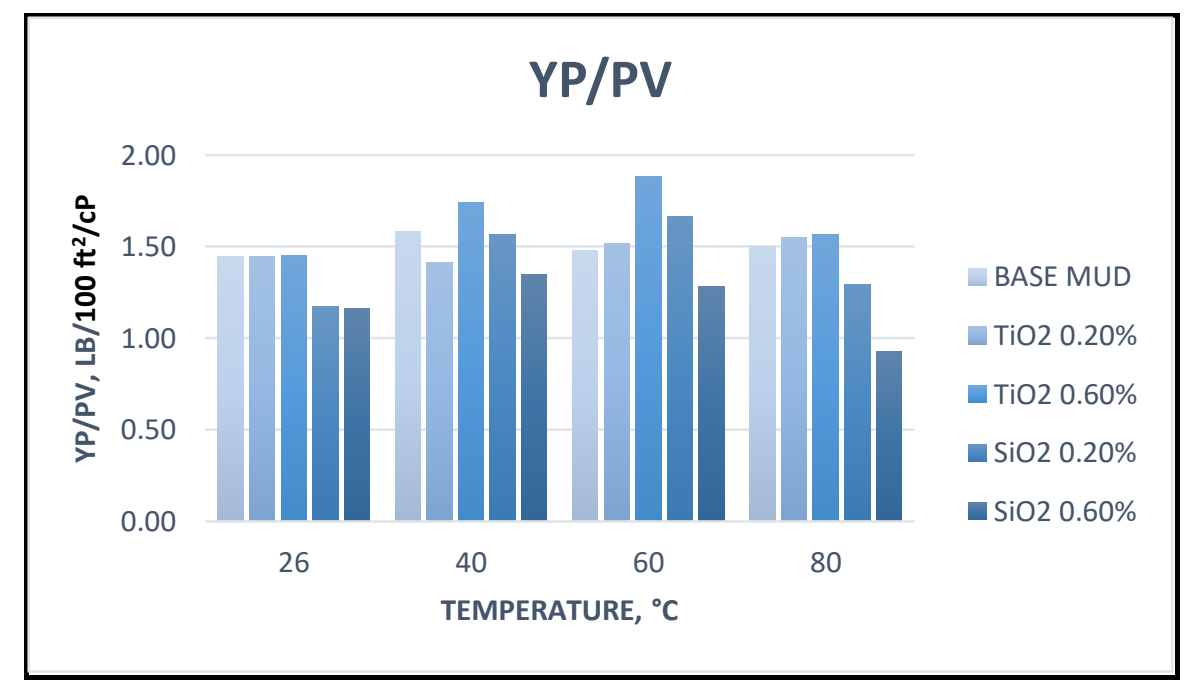

Figure 24: YP/PV relationship before aging

In the case of apparent viscosity (Figure 25), titania nanoparticles had the highest values, while silica nanoparticles had the lowest. Although, this parameter does not give an exact indication of the real viscosity, it is useful to diagnose problems when drilling. It was calculated in the present study by dividing the viscosity reading at 600 RPM by two, so that from the column chart below, it is noticed that the titania nanoparticles showed the highest viscosities at high shear rate. 


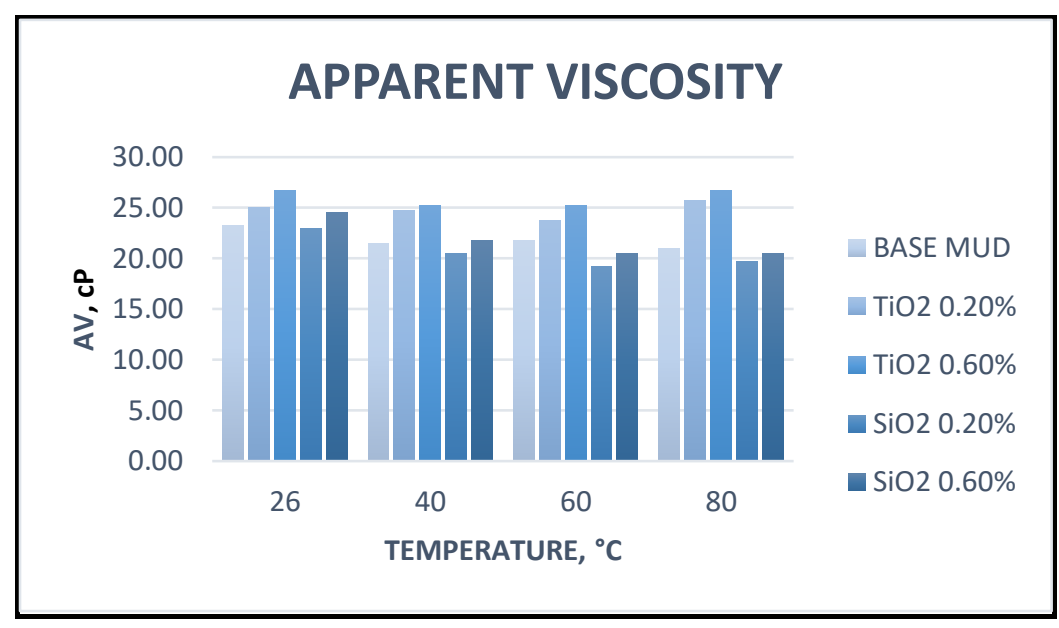

Figure 25: Apparent viscosity before aging

Table 18: Summarizes the analysis for the rheological gel strength in the drilling mud samples and results of the increase in gel strength of mud samples before aging

\begin{tabular}{|c|c|c|c|c|c|}
\hline \multicolumn{6}{|c|}{ RHEOLOGY } \\
\hline \multicolumn{6}{|c|}{ GEL STRENGTH } \\
\hline \multicolumn{6}{|c|}{ BEFORE AGING } \\
\hline $\mathrm{T}\left({ }^{\circ} \mathrm{C}\right)$ & SAMPLE & \multicolumn{2}{|c|}{$10 \mathrm{sec}$} & \multicolumn{2}{|c|}{ INCREASE (\%) } \\
\hline \multirow{5}{*}{26} & BASE MUD & $\square$ & $9.5 \square$ & $14 \sqsubset$ & 47.37 \\
\hline & TiO2 0.20\% & ש & $9.5 \Delta$ & $14.5 \triangle$ & 52.63 \\
\hline & TiO2 0.60\% & $\Delta$ & $12 \triangle$ & $16 \nabla$ & 33.33 \\
\hline & SiO2 $0.20 \%$ & $\nabla$ & $8.5 \nabla$ & $11.5 \nabla$ & 35.29 \\
\hline & $\mathrm{SiO} 20.60 \%$ & $\nabla$ & $9 \nabla$ & $11 \nabla$ & 22.22 \\
\hline \multirow{5}{*}{40} & BASE MUD & $=$ & $9=$ & $14=$ & 55.56 \\
\hline & TiO2 0.20\% & $\Delta$ & $9.5 \Delta$ & $14.5 \nabla$ & 52.63 \\
\hline & TiO2 0.60\% & $\Delta$ & $11 \Delta$ & $16.5 \nabla$ & 50.00 \\
\hline & SiO2 $0.20 \%$ & $\nabla$ & $8 \nabla$ & $10.5 \nabla$ & 31.25 \\
\hline & SiO2 $0.60 \%$ & $\nabla$ & $8 \nabla$ & $10 \nabla$ & 25.00 \\
\hline \multirow{5}{*}{60} & BASE MUD & ש & 7 च & $12 \square$ & 71.43 \\
\hline & TiO2 $0.20 \%$ & $\Delta$ & $8.5 \triangle$ & $14 \nabla$ & 64.71 \\
\hline & TiO2 0.60\% & $\Delta$ & $10 \triangle$ & $16.5 \nabla$ & 65.00 \\
\hline & SiO2 $0.20 \%$ & $\nabla$ & $6 \nabla$ & $8.5 \nabla$ & 41.67 \\
\hline & SiO2 $0.60 \%$ & च & $7 \nabla$ & $9 \nabla$ & 28.57 \\
\hline \multirow{5}{*}{80} & BASE MUD & $=$ & $7=$ & $13=$ & 85.71 \\
\hline & TiO2 0.20\% & $\Delta$ & $9.5 \Delta$ & $16.5 \nabla$ & 73.68 \\
\hline & TiO2 0.60\% & $\Delta$ & $11.5 \Delta$ & $19.5 \nabla$ & 69.57 \\
\hline & $\mathrm{SiO} 20.20 \%$ & $\nabla$ & $6 \nabla$ & $8 \nabla$ & 33.33 \\
\hline & SiO2 $0.60 \%$ & $\nabla$ & $6 \nabla$ & $7.5 \nabla$ & 25.00 \\
\hline
\end{tabular}

In the case of gel strength, the greatest increase at different temperatures was mostly in the base mud, obtaining $85.71 \%$ at $80{ }^{\circ} \mathrm{C}$, as it is plotted in figure 26. However, the readings at 10 seconds and 10 minutes for both concentrations of titania nanoparticles were the largest in magnitude. Silica nanoparticles demonstrated the lowest 
values in both cases. These results indicate that addition of titania in the base mud tends to create a gel structure quicker than the base mud over time. Moreover, this effect was more prominent when the concentration was increased. This may however be beneficial in slowing down the settlement of drill cuttings when mud circulation is stopped in highly deviated wells.

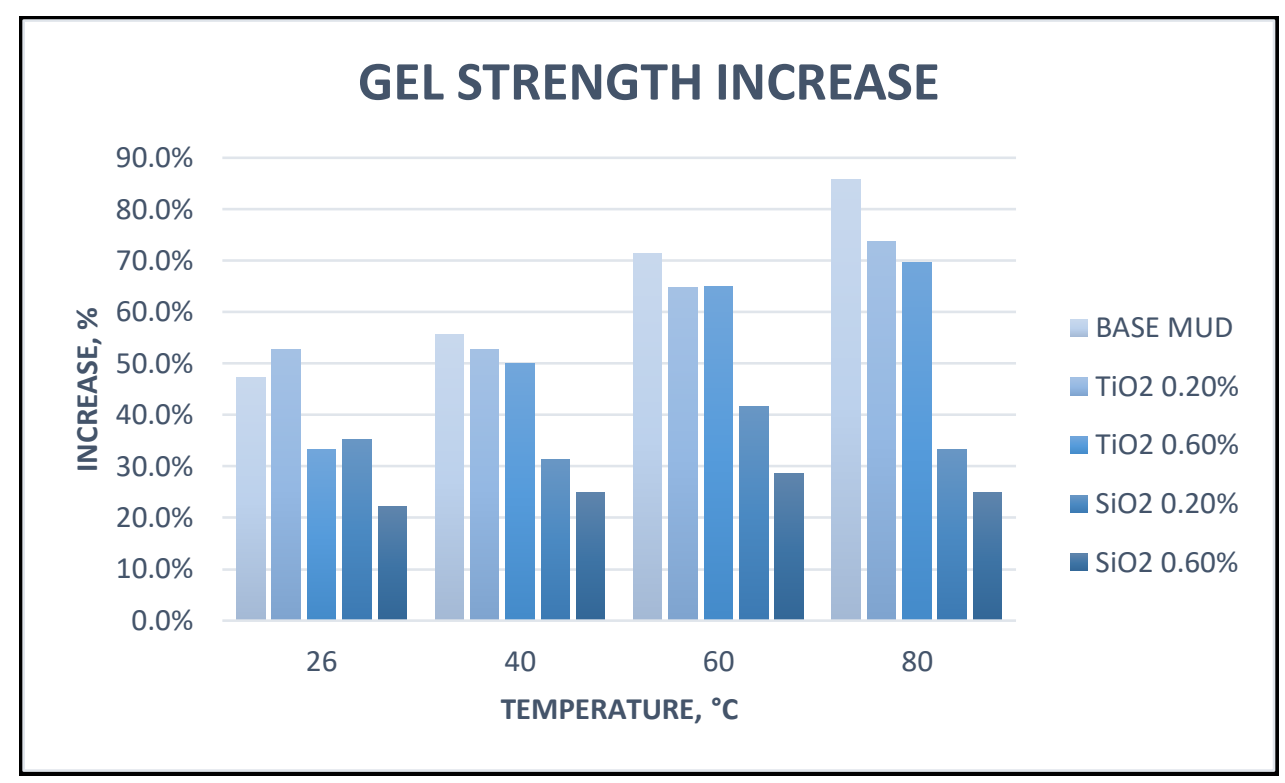

Figure 26: Gel strength increase over time before aging

Silica nanoparticles also showed an increase over time in gel strength parameter when the concentration was increased, although it was minor compared to the other mud samples. In addition, these results indicate that silica nanoparticles could reduce the performance of the drilling mud and induce operational problems, including mechanical sticking of the drill string due to a possible accumulation of drill cuttings. However, silica nanoparticles could prove beneficial in that they could be introduced into the drilling mud to reduce the gel strength of the drilling fluid when it is too high. Regarding gel strength classification, there was insufficient data over time to determine if any of the mud samples showed a progressive or regressive trend. However, it is noteworthy that there is a reduction in the percentage of the gel strength increase when the concentration of nanoparticles is raised. This could indicate that a higher concentration of these nanoparticles produces a slow increment of the gel strength over time. Furthermore, the increase of gel strength on the base mud and titania nanoparticles could be due to a reduction in the water viscosity generated by an increase in temperature, which is also relevant to higher temperature drilling operations (Gray et al. 1980).

\subsection{Rheology-After Aging}

In the post-aging case, the rheology was tested before stirring the mud samples in the mixer and after stirring them. The intention of testing the mud samples before stirring them was to simulate how they could behave once the drill string start to rotate again, after being static for a period. The evaluation after stirring them was done to simulate their behaviour after the drilling mud have been circulated for a period. Two sets of results were therefore obtained.

\subsection{Before Stirring}


Making a comparison between figures 14 and 15, there is a tangible elevation in the viscosity for both concentrations of silica nanoparticles after the aging process before stirring the mud sample. These readings were higher than the mud sample with titania nanoparticles and the base mud. In the case of the mud sample with titania nanoparticles and the base mud, the viscosities decreased after the aging process. This may be attributable to degradation of the other additives (viscosifier agent) in the base mud. The highest increase in plastic viscosity, yield point and apparent viscosity was for silica nanoparticles; in addition, these results were also higher than the readings at the same temperature before aging. This could indicate that after the aging process, flocculation took place and the mechanical friction between particles increased in the drilling mud with silica nanoparticles.

Table 19: Results of PV, YP, YP/PV and AV of mud samples after aging before stirring

\begin{tabular}{|c|c|c|c|c|c|c|c|c|c|}
\hline \multicolumn{10}{|c|}{ RHEOLOGY } \\
\hline \multicolumn{10}{|c|}{$P V$ - YP - YP/PV } \\
\hline \multicolumn{10}{|c|}{ AFTER AGING - BEFORE STIRRING } \\
\hline $\mathrm{T}\left({ }^{\circ} \mathrm{C}\right)$ & SAMPLE & \multicolumn{2}{|c|}{ PV (CP) } & \multicolumn{2}{|c|}{ YP (Ib/100 ft2) } & \multicolumn{2}{|r|}{ YP/PV } & \multicolumn{2}{|c|}{$A V(c p)$} \\
\hline \multirow{5}{*}{26} & BASE MUD & 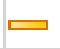 & 12.5 & ש & 14 & 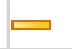 & 1.12 & 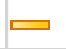 & 19.5 \\
\hline & TiO2 0.20\% & $\Delta$ & 15 & $\Delta$ & 20 & $\Delta$ & 1.33 & $\Delta$ & 25 \\
\hline & TiO2 0.60\% & $\Delta$ & 13.5 & $\Delta$ & 17 & $\Delta$ & 1.26 & $\Delta$ & 22 \\
\hline & SiO2 $0.20 \%$ & $\Delta$ & 16 & $\Delta$ & 22 & $\Delta$ & 1.38 & $\Delta$ & 27 \\
\hline & SiO2 $0.60 \%$ & $\Delta$ & 19 & $\Delta$ & 25.5 & $\Delta$ & 1.34 & $\triangle$ & 31.75 \\
\hline
\end{tabular}

The results for the base mud in Table 19 indicate a decreasing trend in their rheological properties. In the case of yield point, the values indicate that these mud samples may struggle to perform some of their functions, such as carrying drill cuttings, after being static for a long time. A similar deduction may apply to the drilling mud with titania nanoparticles which also exhibit a decreasing trend in yield point. Moreover, the reduction in plastic viscosity of the base mud could be due to a reduction in the mechanical friction of the embedded nano-particles.

Table 20: Results of gel strength of mud samples after aging before stirring

\begin{tabular}{|c|c|c|c|c|c|c|c|}
\hline \multicolumn{8}{|c|}{ RHEOLOGY } \\
\hline \multicolumn{8}{|c|}{ GEL STRENGTH } \\
\hline \multicolumn{8}{|c|}{ AFTER AGING - BEFORE STIRRING } \\
\hline $\mathrm{T}\left({ }^{\circ} \mathrm{C}\right)$ & SAMPLE & & $0 \mathrm{sec}$ & & $0 \mathrm{~min}$ & & NCREASE (\%) \\
\hline \multirow{5}{*}{26} & \multicolumn{3}{|c|}{ BASE MUD $\rightleftharpoons$} & $\square$ & 7 & 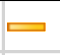 & $40 \%$ \\
\hline & \multicolumn{3}{|c|}{ TiO2 $0.20 \% \triangle$} & $\Delta$ & 9.5 & $\Delta$ & $46 \%$ \\
\hline & \multicolumn{3}{|c|}{$\mathrm{TiO} 20.60 \% \triangle$} & $\Delta$ & 9 & $\nabla$ & $29 \%$ \\
\hline & \multicolumn{3}{|c|}{ SiO2 $0.20 \% \triangle$} & $\Delta$ & 7.5 & $\nabla$ & $25 \%$ \\
\hline & \multicolumn{3}{|c|}{ SiO2 $0.60 \% \triangle$} & $\Delta$ & 9 & $\nabla$ & $13 \%$ \\
\hline
\end{tabular}

In the case of gel strength, all the results showed a poor performance, as can be observed in Table 20. It is also apparent however that the increase in gel strength in silica nanoparticles compared to the base mud, was lower before the aging process. However, due to the low results in gel strength, it would appear that the mud samples are not able to suspend drill cuttings after being static at borehole conditions over a long period which could cause operational issues.

\subsection{After Stirring}


After stirring the mud samples, it can be observed at figure 15 that the viscosities readings during different shear rates decreased further, for all the mud samples. However, a similar trend to the previous case is observed; the highest viscosities readings were obtained with $0.60 \%(\mathrm{w} / \mathrm{w}) \mathrm{SiO}_{2}$, and the lowest were for the base mud without any nano-particles.

Table 21: Results of PV, YP, YP/PV and AV of mud samples after aging after stirring

\begin{tabular}{|c|c|c|c|c|c|c|c|c|c|}
\hline \multicolumn{10}{|c|}{ RHEOLOGY } \\
\hline \multicolumn{10}{|c|}{ PV - YP - AV } \\
\hline \multicolumn{10}{|c|}{ AFTER AGING - AFTER STIRRING } \\
\hline $\mathrm{T}\left({ }^{\circ} \mathrm{C}\right)$ & SAMPLE & \multicolumn{2}{|c|}{$\mathrm{PV}(\mathrm{CP})$} & \multicolumn{2}{|c|}{ YP (lb/100 ft2) } & \multicolumn{2}{|r|}{ YP/PV } & \multicolumn{2}{|c|}{ AV (cp) } \\
\hline \multirow{5}{*}{26} & BASE MUD & $\square$ & 8.5 & $\square$ & 9.5 & $\square$ & 1.12 & $\square$ & 13.25 \\
\hline & TiO2 0.20\% & $\Delta$ & 10.5 & $\Delta$ & 13.5 & $\Delta$ & 1.29 & $\Delta$ & 17.25 \\
\hline & TiO2 0.60\% & $\Delta$ & 10 & $\Delta$ & 12 & $\Delta$ & 1.20 & $\Delta$ & 16 \\
\hline & SiO2 $0.20 \%$ & $\Delta$ & 11 & $\Delta$ & 11 & $\nabla$ & 1.00 & $\Delta$ & 16.5 \\
\hline & $\mathrm{SiO} 20.60 \%$ & $\Delta$ & 12.5 & $\Delta$ & 13.5 & $\nabla$ & 1.08 & $\Delta$ & 19.25 \\
\hline
\end{tabular}

Table 22: Results of gel strength of mud samples after aging after stirring

\begin{tabular}{|c|c|c|c|c|c|c|c|}
\hline \multicolumn{8}{|c|}{ RHEOLOGY } \\
\hline \multicolumn{8}{|c|}{ GEL STRENGTH } \\
\hline \multicolumn{8}{|c|}{ AFTER AGING - AFTER STIRRING } \\
\hline $\mathrm{T}\left({ }^{\circ} \mathrm{C}\right)$ & SAMPLE & \multicolumn{2}{|c|}{$10 \mathrm{sec}$} & \multicolumn{2}{|c|}{$10 \mathrm{~min}$} & \multicolumn{2}{|c|}{ INCREASE (\%) } \\
\hline \multirow{5}{*}{26} & BASE MUD & 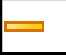 & 4 & ש & 7 & ש & $75 \%$ \\
\hline & TiO2 0.20\% & $\triangle$ & 4.5 & 二 & 7 & $\nabla$ & $56 \%$ \\
\hline & TiO2 0.60\% & $\triangle$ & 5 & $\Delta$ & 8 & $\nabla$ & $60 \%$ \\
\hline & SiO2 $0.20 \%$ & $\triangle$ & 4.5 & $\nabla$ & 6 & $\nabla$ & $33 \%$ \\
\hline & SiO2 $0.60 \%$ & $\Delta$ & 5 & 二 & 7 & $\nabla$ & $40 \%$ \\
\hline
\end{tabular}

Tables 21 and 22 shows that there were not a favourable trend on rheological properties of the drilling mud samples after stirring them in the mixer. In fact, all the results were lower than the rheological tests before stirring. This loss in viscosity may be directly attributable to the degradation of the viscosifier agent during the aging process.

\subsection{Lubricity}

The results obtained during the lubricity test indicated that titania nanoparticles at a concentration of $0.60 \%$ (w/w) gave the best performance for torque compared to the other mud samples, although, a large decrease in torque was not observed. However, taking into account that the torque readings in the base mud tended to increase over time before and after aging, these results could be relevant. To evaluate the lubricity of the mud samples, the CoF was calculated using equation (4). This factor provides a more accurate value for the trend of the lubricity during the tests since it incorporates a correction factor. For the case of the base mud, the CoF for the base mud is calculated as follows:

$$
\mathrm{CoF}_{\text {Base Mud }\left(26^{0} \mathrm{c}, 1 \mathrm{~min}\right)}=\frac{37.5 \mathrm{in}-\text { pounds } \times 0.94}{100 \mathrm{lbf}}
$$


CoF

The same calculation was performed for the rest of the mud samples. We consider again both cases before aging and after aging.

\subsection{Before Aging}

From the results of the lubricity test, which can be observed from Tables 10 to 14 and from figures 16-20, it can be appreciated that the results of titania nanoparticles at a concentration of $0.60 \%(\mathrm{w} / \mathrm{w})$ showed a decreasing trend (and the lowest values) for the torque readings at different temperatures. However, at a lower concentration $(0.20 \% \mathrm{w} / \mathrm{w})$, they showed the highest torque readings. In the case of silica nanoparticles, at a concentration of $0.20 \%(\mathrm{w} / \mathrm{w})$, the torque readings were close to the base mud and showed a stable growth over time. However, when their concentration was raised, the torque also increased; however, these readings decreased over time, although, they were not lower than the results of the base mud. Table 23 shows the variation in percentage of the torque readings of the treated base mud with nanoparticles relative to the untreated base mud. Here (and also in

Table 24), a negative value means a reduction and a positive value means an increase, moreover, the results are highlighted with a blue colour scale which gets darker with an increase in the percentage.

Table 23: Percentage in torque variation before aging

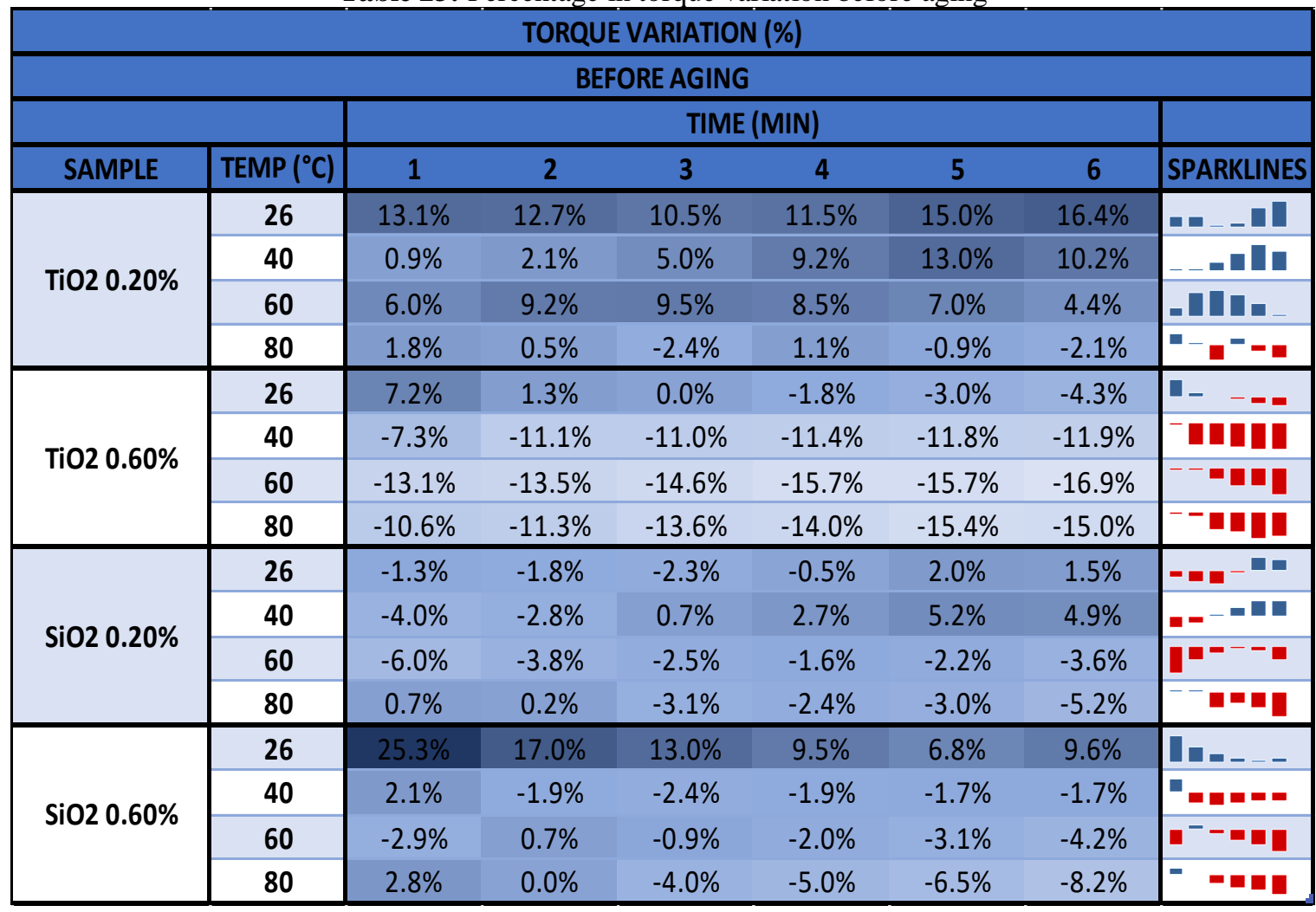

In Table 23 the highest decrease in percentage was for titania nanoparticles at $0.60 \%(\mathrm{w} / \mathrm{w})$ and the torque percentage tended to reduce with an increase in temperature, as well as, silica nanoparticles at $0.60 \%$ (w/w). Moreover, titania nanoparticles at $0.20 \%(\mathrm{w} / \mathrm{w})$ and silica nanoparticles at $0.20 \%(\mathrm{w} / \mathrm{w})$, both showed a decreasing trend from $60{ }^{\circ} \mathrm{C}$, although both of them show some fluctuation. Regarding the CoF analysis, table 24 shows the results for the torque readings at different temperatures using the same format as table 23. 
Table 24: Coefficient of friction before aging

\begin{tabular}{|c|c|c|c|c|c|c|c|c|c|}
\hline \multicolumn{10}{|c|}{$\mathrm{COF}$} \\
\hline \multicolumn{10}{|c|}{ BEFORE AGING } \\
\hline & & \multicolumn{8}{|c|}{ TIME } \\
\hline SAMPLE & $\operatorname{TEMP}\left({ }^{\circ} \mathrm{C}\right)$ & 1 & 2 & 3 & 4 & 5 & 6 & SPARKLINES & AVERAGE \\
\hline \multirow{4}{*}{ BASE MUD } & 26 & 0.354 & 0.373 & 0.378 & 0.378 & 0.377 & 0.374 & - & 0.37 \\
\hline & 40 & 0.399 & 0.400 & 0.396 & 0.391 & 0.384 & 0.388 & प⿴囗十_- & 0.39 \\
\hline & 60 & 0.427 & 0.419 & 0.419 & 0.421 & 0.421 & 0.425 & $\square_{--ー ー \square}$ & 0.42 \\
\hline & 80 & 0.412 & 0.419 & 0.430 & 0.432 & 0.435 & 0.440 & -_E口ص & 0.43 \\
\hline \multirow{4}{*}{ TiO2 0.20\% } & 26 & 0.400 & 0.420 & 0.417 & 0.421 & 0.433 & 0.435 & 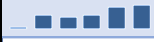 & 0.42 \\
\hline & 40 & 0.403 & 0.409 & 0.416 & 0.427 & 0.434 & 0.428 & - - EDᄆ & 0.42 \\
\hline & 60 & 0.452 & 0.458 & 0.459 & 0.457 & 0.450 & 0.444 & ㅁㅁㅁㄷㅡ. & 0.45 \\
\hline & 80 & 0.419 & 0.421 & 0.419 & 0.437 & 0.431 & 0.430 & $---\square \square$ & 0.43 \\
\hline \multirow{4}{*}{ TiO2 0.60\% } & 26 & 0.379 & 0.378 & 0.378 & 0.371 & 0.365 & 0.358 & 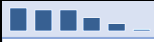 & 0.37 \\
\hline & 40 & 0.370 & 0.356 & 0.353 & 0.346 & 0.339 & 0.342 & 口曰ローーー & 0.35 \\
\hline & 60 & 0.371 & 0.362 & 0.358 & 0.355 & 0.355 & 0.353 & 口Eーーー- & 0.36 \\
\hline & 80 & 0.368 & 0.372 & 0.371 & 0.372 & 0.368 & 0.374 & _ㅁㅁㅁㅁ들 & 0.37 \\
\hline \multirow{4}{*}{ SiO2 $0.20 \%$} & 26 & 0.349 & 0.366 & 0.369 & 0.376 & 0.384 & 0.379 & - & 0.37 \\
\hline & 40 & 0.383 & 0.389 & 0.399 & 0.401 & 0.404 & 0.407 & - - & 0.40 \\
\hline & 60 & 0.401 & 0.403 & 0.409 & 0.414 & 0.412 & 0.410 & - - & 0.41 \\
\hline & 80 & 0.414 & 0.420 & 0.416 & 0.422 & 0.422 & 0.417 & 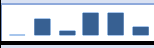 & 0.42 \\
\hline \multirow{4}{*}{ SiO2 0.60\% } & 26 & 0.444 & 0.436 & 0.427 & 0.413 & 0.402 & 0.410 & DEn-ー- & 0.42 \\
\hline & 40 & 0.408 & 0.393 & 0.387 & 0.383 & 0.378 & 0.381 & 口aーーーー & 0.39 \\
\hline & 60 & 0.414 & 0.422 & 0.415 & 0.413 & 0.408 & 0.407 & ㅂㅁㅂ_- & 0.41 \\
\hline & 80 & 0.423 & 0.419 & 0.413 & 0.411 & 0.407 & 0.404 & 口na=ー- & 0.41 \\
\hline
\end{tabular}

A similar trend for the mud samples with nanoparticles is evident as in the case of table 23. Moreover, an increasing trend in the CoF of the base mud with a rise of temperature is clearly evident in Table 24 . These tables imply that the addition of silica nanoparticles in the base mud tends to improve the lubricity, even with a small concentration. In addition, these trends suggest that it could be necessary to increase the nano-particle concentration to achieve better results, since the maximum reduction in torque was about $8 \%$. However, in the case of titania nanoparticles the highest reduction in torque was about $16 \%$ (double that of silica nano-particles). The reduction in torque and CoF of titania nanoparticles at $0.60 \%(\mathrm{w} / \mathrm{w})$ would also suggest that an increase in their concentration could further depress both parameters, with the implication that titania nanoparticles could have a greater impact in the improvement of drilling mud lubricity than silica nanoparticles. Lubricity refers to the reduction in friction and or wear by a lubricant and therefore the presence of titania nano-particles offers clear tribological advantages. The tables 23 and 24 further show that the torque and CoF of the base mud increases with temperature, whereas the addition of nanoparticles in the base mud shows the contrary effect. The reduction is more significant with a higher concentration of titania nanoparticles and this may also be evidence for the improved thermal conductivity (and therefore heat dissipation characteristic) of nanoparticles, which has been emphasized in numerous experimental studies including (Choi, 1995; Hung and Gu, 2014) Due to the presence of nanoparticles around the lubricating film created between the lubricity ring and block in the lubricity tests, the nano-particles may have isolated and dissipated the heat more efficiently around this film. Silica and titania are both ceramic materials which are known to have excellent thermal dissipation properties. As nano-particles these materials retain this feature.

\subsection{After Aging}

In this case, the lubricity test was carried out only after stirring the mud samples and cooling them to recreate the lubricity behaviour representative of drilling muds after circulation for some time after following a period in which 
they are static at the bottom hole condition. From table 15 and figure 21 (section 3) it can be observed that the results were lower than the previous test at the same temperature (before the aging process), although, the growing trend exhibited by the base mud remained. However, the other mud samples did not exhibit the same pattern. For titania and silica nanoparticles at $0.60 \%(\mathrm{w} / \mathrm{w})$ the trend changed and remained stable with slight fluctuations, while titania and silica at $0.20 \%(\mathrm{w} / \mathrm{w})$ showed a decreasing trend. Below, table 25 shows the torque variation in percentage after the aging process compared to the base mud.

Table 25: Percentage in torque variation after aging

\begin{tabular}{|c|c|c|c|c|c|c|c|c|}
\hline \multicolumn{9}{|c|}{ TORQUE VARIATION (\%) } \\
\hline \multicolumn{9}{|c|}{ AFTER AGING } \\
\hline & & \multicolumn{6}{|c|}{ TIME (MIN) } & \\
\hline TEMP $\left({ }^{\circ} \mathrm{C}\right)$ & SAMPLE & 1 & 2 & 3 & 4 & 5 & 6 & SPARKLINES \\
\hline \multirow{4}{*}{26} & TiO2 0.20\% & $30.4 \%$ & $8.8 \%$ & $7.9 \%$ & $3.7 \%$ & $5.0 \%$ & $5.3 \%$ & $-\ldots-\ldots$ \\
\hline & TiO2 $0.60 \%$ & $18.2 \%$ & $2.1 \%$ & $-1.5 \%$ & $-2.9 \%$ & $-1.7 \%$ & $-0.6 \%$ & ----- \\
\hline & SiO2 0.20\% & $44.3 \%$ & $17.9 \%$ & $10.9 \%$ & $6.6 \%$ & $8.7 \%$ & $8.2 \%$ & $-\ldots-\ldots$ \\
\hline & SiO2 $0.60 \%$ & $33.9 \%$ & $16.7 \%$ & $11.4 \%$ & $10.3 \%$ & $12.0 \%$ & $11.4 \%$ & ---- \\
\hline
\end{tabular}

In this scenario, the torque variation for all the mud samples with nanoparticles showed a considerable drop after the first minute of the test, and then, it was followed by a moderate fall over time. However, in most samples, the reduction was not below than the torque readings of the base mud; only titania nanoparticles at $0.60 \%$ (w/w) were below the base mud values. Finally, Table 26 shows the results of the calculation of the CoF after aging. In general, the results follow the same trend as the torque readings of this test. The friction factors of the mud with nanoparticles were close to the base mud friction factor.

Table 26: Coefficient of friction after aging

\begin{tabular}{|c|c|c|c|c|c|c|c|c|c|}
\hline \multicolumn{10}{|c|}{ COF } \\
\hline \multicolumn{10}{|c|}{ AFTER AGING } \\
\hline \multicolumn{10}{|c|}{ TIME (MIN) } \\
\hline TEMP $\left({ }^{\circ} \mathrm{C}\right)$ & SAMPLE & 1 & 2 & 3 & 4 & 5 & 6 & SPARKLINES & AVERAGE \\
\hline \multirow{5}{*}{26} & BASE MUD & 0.263 & 0.309 & 0.321 & 0.327 & 0.322 & 0.321 & - & 0.311 \\
\hline & TiO2 0.20\% & 0.343 & 0.337 & 0.346 & 0.339 & 0.338 & 0.337 & $\square-\square---$ & 0.340 \\
\hline & TiO2 0.60\% & 0.311 & 0.316 & 0.316 & 0.318 & 0.317 & 0.319 & 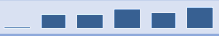 & 0.316 \\
\hline & SiO2 $0.20 \%$ & 0.380 & 0.365 & 0.355 & 0.349 & 0.351 & 0.347 & $a=--\ldots$ & 0.358 \\
\hline & SiO2 $0.60 \%$ & 0.353 & 0.361 & 0.357 & 0.361 & 0.361 & 0.357 & $\square=\square[$ & 0.358 \\
\hline
\end{tabular}

In general, the results of the lubricity after the aging process demonstrated the best performance for all the mud samples. All of these results showed a considerable reduction in the CoF compared to the results before aging. However, only titania nanoparticles at $0.60 \%(\mathrm{w} / \mathrm{w})$ were below the friction factor of the base mud over time, although, only a marginal decrease was achieved. Further experiments are warranted to evaluate the lubricity of the mud samples at different temperatures both before and after aging to confirm if these nanoparticles still could contribute to isolating heat in the lubricating film and show a decreasing trend.

\section{CONCLUSIONS}

An experimental study has been described to evaluate the rheological characteristics of water based muds using nano-particles. Five mud samples were examined, namely ordinary base mud, two cases of titania nano-particle doped water based mud and two cases of silica nano-particle doped base mud. Density, rheology (gel strength, apparent viscosity, shear stress) and lubricity (torque and coefficient of friction) properties have been quantified using a variety of devices. Both before aging and after aging scenarios have been studied and the influence of 
temperature in particular on rheology and lubricity characteristics examined in detail. The pertinent conclusions from the present study may be summarized as follows:

- A small enhancement in base mud density is caused with silica nanoparticles with no tangible modification achieved with titania nanoparticles. Before aging, titania nanoparticles achieve greater viscosity than base mud or silica-doped mud which reduces viscosity.

- Before aging, titania nanoparticles result in higher plastic viscosity (PV), yield point (YP) and apparent viscosity (AV) of the drilling mud, although a decreased yield point when the concentration is lower i.e. $0.20 \%$ (w/w) at $40{ }^{\circ} \mathrm{C}$.

- Before aging, silica nanoparticles generally result in a decrease in the yield point and apparent viscosity of the drilling mud, which may be related to super hydrophilic characteristics.

- Before aging, titania nanoparticles achieve the highest efficiency (low plastic viscosity and a high yield point) whereas silica nanoparticles showed the lowest efficiency.

- Before aging, higher values of gel strength are associated with titania and lower values with silica nanoparticles, whereas the greatest increase in gel strength is with base mud. The introduction of titania in the base mud generates a gel structure quicker than the base mud over time.

- After aging, and before stirring, greater plastic and apparent viscosity is achieved for silica nanoparticles and lower viscosities with the titania nano-particles.

- After aging and before stirring, titania nanoparticles cause a reduction in the drilling mud yield point whereas silica nano-particles cause the opposite effect.

- After aging and before stirring, the increase in gel strength in silica nanoparticles relative to the base mud is less dramatic than before aging.

- After aging and stirring, the greatest viscosities are achieved with $0.60 \%(\mathrm{w} / \mathrm{w}) \mathrm{SiO}_{2}$, and the lowest are associated with the base mud without any nano-particles.

- Before aging, with regard to lubricity, titania nanoparticles at a concentration of $0.60 \%(\mathrm{w} / \mathrm{w})$ attained the best performance for torque (i.e. lowest torque) compared to the other mud samples, although, a significant reduction in torque was not measured.

- Before aging, silica nanoparticles, at a concentration of $0.20 \%(\mathrm{w} / \mathrm{w})$, produced torque readings close to those of the base mud although these values increased with time i.e. worst torque performance.

- Before aging, torque and $\mathrm{CoF}$ of the base mud increases with temperature, whereas the addition of nanoparticles in the base mud results in a decrease in torque and CoF with temperature indicating an increase in heat dissipation (improved thermal conductivity) with nanoparticles.

- After aging, only titania nanoparticles at $0.60 \%(\mathrm{w} / \mathrm{w})$ achieved CoF magnitudes below the base mud values indicating a non-trivial enhancement in lubricity.

- In general, the results of the lubricity after the aging process were superior to those before aging.

The results generally indicate that based on properties measured before the aging process, titania nanoparticles at a concentration of $0.60 \%(\mathrm{w} / \mathrm{w})$ could be used as an additive to improve the rheological properties and lubricity of a drilling fluid, provided a stable formulation is achievable for long periods of time. In this regard they offer some promise as robust additives for reducing the torque and drag in highly deviated and ERD wells where there is no presence of shale. Further investigations are required however to gain deeper insight into the performance 
of both silica and titania (and indeed other) nano-particles in order to confirm their potential benefits for drilling operations. The present investigation also provides some useful and much-needed rheological data for numerical and mathematical models and efforts for calibrating such models with computational fluid dynamics codes are presently under way. Furthermore, there is an extensive range of other metallic nano-particles which could be explored including silver, zinc, copper etc. Also, further studies must be conducted e.g. field trials to establish if indeed the nano-particles work in full scale operations. It is hoped that the present work will stimulate petroleum engineers working in the field to consider this.

\section{ACKNOWLEDGEMENTS}

The authors are grateful to the reviewers for their insightful comments which have served to significantly improve the present article.

\section{FUNDING}

This research did not receive any specific grant from funding agencies in the public, commercial, or not- for profit sectors.

\section{DECLARATION OF CONFLICTS OF INTEREST}

The author(s) declared no potential conflicts of interest with respect to the research, authorship, and/or publication of this article.

\section{REFERENCES}

Abdo J, Haneef MD (2013) Clay nanoparticles modified drilling fluids for drilling of deep hydrocarbon wells. Appl Clay Sci 86: 76-82.

Ahmet S, Mustafa VK, Reha O (2013) Performance analysis of drilling fluid liquid lubricants. J Petroleum Sci Eng 108: 64-73.

Al-Yasiri MS, Al-Sallami WT (2015) How the drilling fluids can be made more efficient by using nanomaterials. American J Nano-Research and Applications 3:41-45.

Amanullah Md, Al-Arfaj MK, Al-Abdullatif Z (2011) Preliminary test results of nano-based drilling fluids for oil and gas field application. SPE Drilling Conference and Exhibition, Amsterdam, The Netherlands, March 1-3 SPE/IADC 139534. doi:10.2118/139534-MS

Baker Hughes (1995) INTEQ., Drilling Engineering Workbook. Baker Hughes INTEQ, Houston, Texas, USA.

Bég O. Anwar, Basir MFM, Uddin MJ, Ismail AIM (2017) Numerical study of slip effects on asymmetric bioconvective nanofluid flow in a porous microchannel with an expanding/contracting upper wall using Buongiorno's model. J Mechanics in Medicine and Biology 17(5): 1750059 (28 pages) doi: 10.1142/S0219519417500592.

Behnamanhar H, Noorbakhsh SS, Maghsoudloojafari H (2014) Environmentally friendly water-based drilling fluid for drilling of water-sensitive formations. J Petroleum and Gas Exploration Research 4(4): 6071. 
Bu H, Sun J, Wang C, Bu P (2013) Rheological properties of polymer-gel drilling fluids at high temperature and pressure. Chemistry and Technology of Fuels and Oils 48 (6): 449-458.

Choi SUS (1995) Enhancing thermal conductivity of fluids with nanoparticles. Development of Applications of non-Newtonian Flows. ASME J Fluids Eng. 66:99-105.

Dantas APT, Leite RS, Nascimento RCAM, Amorim LV (2014) The influence of chemical additives in filtration control of inhibited drilling fluids. Brazilian Journal of Petroleum and Gas 8(3): 097-108.

Dingsøyr E, Pedersen E, Taugbøl K (2004) Oil Based drilling fluids with tailor-made rheological properties: results from a multivariate analysis. Annual Transactions of the Nordic Rheology Society 12: 10-23.

FANN (2016) Retrieved from http://www.fann.com/fann/products/supplies-and-reagents/lab-equipmentsupplies/blenders-and-mixers/mixers-hb.page.

Fazelabdolabadi B, Khodadadi AA, Sedaghatzadeh M (2015) Thermal and rheological properties improvement of drilling fluids using functionalized carbon nanotubes. Applied Nanoscience_5(6): 651-659.

Gray GR, Darley HCH, Rogers WF (1980) Composition and Properties of Oil Well Drilling Fluids. Gulf Publishing Company, Houston, Texas, USA.

Huminic G, Huminic A (2012) Application of nanofluids in heat exchangers: A review. Renewable and Sustainable Energy Reviews 16:5625-5638.

Hung YH, Gu HJ (2014) Multiwalled carbon nanotube nanofluids used for heat dissipation in hybrid green energy systems. J Nanomaterials, Article ID: 196074 (12 Pages). doi:10.1155/2014/196074.

Hwang Y, Lee J, Lee J, Jeong Y, Cheong S, Ahn Y, Kim S (2008) Production and dispersion stability of nanoparticles in nanofluids. Powder Tech.186(2):145-153.

Kaloudis E, Papanicolaou E, Belessiotis V (2016) Numerical simulations of a parabolic trough solar collector with nanofluid using a two-phase model. Renewable Energy 97:218-229.

Kannaiyan K, Anoop K, Sadr R (2016) Effect of nanoparticles on the fuel properties and spray performance of aviation turbine fuel. ASME J. Energy Resources Tech. 139(3):032201-032201-8.

Lin Jin, Dan Yue, Zhe-Wu Xu, Guobin Liang, Yilei Zhang, Jian-Fu Zhang, Xingcai Zhang and Zhenling Wang (2014) Fabrication, mechanical properties, and biocompatibility of reduced graphene oxide-reinforced nanofiber mats, RCS Adv. 4(66) 303-35041.

Mostafavi V, Ferdous MZ, Hareland G, Husein M (2011) Design and application of novel nano drilling fluids to mitigate circulation loss problems during oil well drilling operations. Clean Tech 383-386.

OFITE (2014) OFI TESTING EQUIPMENT, INC. Retrieved from http://www.ofite.com/products/product/43mud-balance-4-scale-metal.

Olatunde AO, Usman MA, Olafadehan OA, Adeosun TA, Ufot OE, Improvement of rheological properties of drilling fluid using locally based materials. Petroleum \& Coal 54(1): 65-75.

Qu Y, Lai X, Zou L, Su Y (2009) Polyoxyalkyleneamine as shale inhibitor in water-based drilling fluids. Applied Clay Science 44(3): 265-268.

Shuang Yang, Yu Hou, Bo Zhang, Xiao Hua Yang, Haimin Zhang, Hui Jun Zhao and Hua Gui Yang (2014) Precisely controlled heterogeneous nucleation sites for $\mathrm{TiO}_{2}$ crystal growth, Cryst Eng Comm, 16, 7502-7506.

Shuang Yang, Nian Huang, Yong Mei Jin, Hui Qing Zhang, Yong Hua Su and Hua Gui Yang (2015) Crystal shape engineering of anatase $\mathrm{TiO}_{2}$ and its biomedical applications. Cryst Eng Comm, 17, 6617-6631 
Slawomir R, Zbigniew KB, Malgorzata U (2009) Study on the application of starch derivatives as the regulators of potassium drilling fluids filtration. Chemistry and Chemical Tech 3:107-202.

Ying Tian, Xingcai Zhang, Hong-Zhang Geng, Hai-Jie Yang, Chungang Li, Shi-Xun Da, Xiushan Lu, Jie Wang and Song-Lin Jia (2017) Carbon nanotube/polyurethane films with high transparency, low sheet resistance and strong adhesion for antistatic application, RSC Adv., 7(83), 53018-53024.

Yuri M. Lvov, Pravin Pattekari, Xingcai Zhang, Vladimir Torchilin, Converting poorly soluble materials into stable aqueous nanocolloids, Langmuir 27 (3), 1212-1217 (2011).

Zakaria M, Husein MM, Harland G (2012) Novel nanoparticle-based drilling fluid with improved characteristics, SPE International Oilfield Nanotechnology Conference and Exhibition, 12-14 June, Noordwijk, The Netherlands.

\section{APPENDIX}

Properties of nano-particles used in the experiments

Table A1 Properties of Titanium Oxide $\mathrm{Ti}_{2}$ Nanoparticles (US Research Nanomaterials, Inc, 2016).

\begin{tabular}{|c|c|}
\hline \multicolumn{2}{|c|}{ Titanium Oxide (Ti 02) Nanoparticles } \\
\hline \multicolumn{2}{|c|}{ Properties } \\
\hline Purity & $99.9 \%$ \\
\hline Average Particle Size & $30 \mathrm{~nm}$ \\
\hline Specific Surface Area & $35-60 \mathrm{~m}^{2} / \mathrm{gr}^{|c|}$ \\
\hline Colour & White \\
\hline Morphology & Near Spherical \\
\hline Bulk Density & $0.25 \mathrm{gr} / \mathrm{cm}^{3}$ \\
\hline True Density & $4.23 \mathrm{gr} / \mathrm{cm}^{3}$ \\
\hline
\end{tabular}

Table A2: Properties of Silicon Dioxide $\mathrm{Si}_{2}$ Nanoparticles (US Research Nanomaterials, Inc, 2016)

\begin{tabular}{|c|c|}
\hline \multicolumn{2}{|c|}{ Silicon Dioxide (Si02) Nanoparticles } \\
\hline \multicolumn{2}{|c|}{ Properties } \\
\hline Purity & $96.3 \%$ \\
\hline Average Particle Size & $20-30 \mathrm{~nm}$ \\
\hline Specific Surface Area & $130-600 \mathrm{~m}^{2} / \mathrm{gr}$ \\
\hline Colour & White \\
\hline Bulk Density & $<0.10 \mathrm{gr} / \mathrm{cm}^{3}$ \\
\hline True Density & $2.4 \mathrm{gr} / \mathrm{cm}^{3}$ \\
\hline
\end{tabular}

